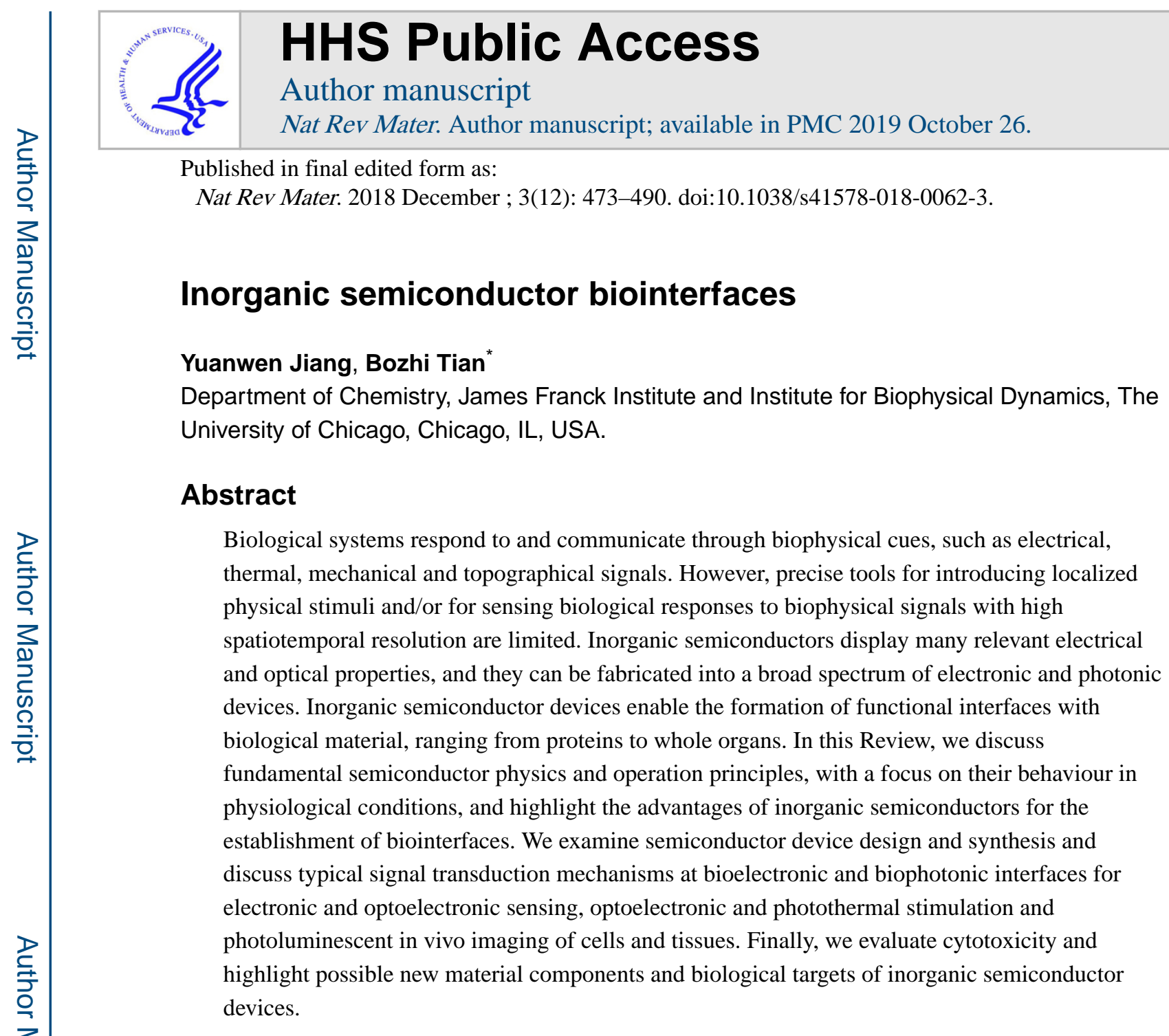

Inorganic semiconductors display a diverse spectrum of physical properties and thus can be fabricated as device platforms for a variety of applications ${ }^{1-8}$, for example, for electronics, photonics and energy conversion. Compared with polymers and metals, semiconductors are especially applicable for electronic and photonic biointerface studies because they can be configured into multifunctional devices (for example, sensors, modulators and switches) with desired properties (for example, amplification, fast signal transduction and low power consumption $)^{6-8}$.

Organic semiconductors also possess several relevant features ${ }^{9,10}$, such as improved mechanical compliance, easier solution processability and intrinsic stretchability through molecular designs, compared with inorganic semiconductors and metals. However, inorganic materials offer certain advantages, in particular, diverse signal transduction mechanisms at a biointerface. High charge carrier mobilities in inorganic materials and correspondingly fast

\footnotetext{
*btian@uchicago.edu.

Author contributions

Y.J. and B.T. wrote the paper.

Competing interests

The authors declare no competing interests.
} 
responses and high sensitivity in inorganic devices enable more accurate probing of complex biological dynamics than can be achieved by organic materials. Moreover, high-performance inorganic semiconductors can be precisely fabricated into various architectures at the nanoscale, matching the size of subcellular and molecular components. Compared with organic electronics and optoelectronics, inorganic devices usually require lower operation voltages, allowing easier device passivation in physiological fluids. Finally, the operation of inorganic semiconductor devices typically does not involve mobile ions in the interior of a device, and therefore, dry-state device physics and semiconductor surface control can be readily applied to biointerfaces ${ }^{7,8}$.

Since the invention of the field-effect transistor (FET) in the late $1940 \mathrm{~s}^{11}$, semiconductor materials and devices have become a key component of electronics technology. Progress in device scalability and electronic and ionic signal transduction, and the possibility of remotely controlled processes have enabled the engineering of integrated circuits ${ }^{12}$, ionsensitive FETs ${ }^{13}$ and light-addressable cellular sensors ${ }^{14}$ (FIG. 1). The development of biocompatible and biodegradable semiconductor materials ${ }^{15,16}$, optical labelling ${ }^{17,18}$ and deformable and stretchable devices ${ }^{16,19}$ facilitated the application of semi-conductor devices in biology, for example, to establish electrical ${ }^{16,20-22}$ and optical ${ }^{23,24}$ interfaces with neural systems, for nano-bioelectronics ${ }^{1,25,26}$, in semiconductor-enabled synthetic biology ${ }^{27-29}$, in biodegradable semiconductor implants ${ }^{16}$ and for the non-genetic optical control of animal motion ${ }^{30}$. The design of a neuron-silicon junction, at which single leech neurons (Retzius cells) can establish a biointerface at the open gate of a p-type $\mathrm{FET}^{20}$, is one such example. In this setup, the transistor can record both spontaneous and stimulated neural activities. The first semiconductor-based intracellular electrical recording was achieved using a flexible and movable nanoscale $\mathrm{FET}^{31}$. In this device, the semiconductor component was synthesized by gold-nanoparticle-catalysed chemical vapour deposition.

Inorganic-semiconductor-based biointerfaces and biophysical studies have become increasingly important for the investigation of cellular biophysics, for example, for electronic sensing and optoelectronic stimulation, in synthetic biology and artificial intelligence and for initiatives such as the Brain Research through Advancing Innovative Neurotechnologies (BRAIN) initiative ${ }^{32,33}$.

In this Review, we discuss inorganic semiconductors for bioelectronic and biophotonic interfaces. We highlight important aspects of semiconductor physics at biointerfaces under physiological conditions and give an overview of semiconductor devices, including 0D, 1D, 2D and 3D semiconductor configurations, chemical procedures and semiconductor degradation behaviours. Finally, we highlight key biophysical mechanisms underlying bioelectronic and biophotonic interfaces and investigate the cytotoxicity of semiconductors.

\section{Semiconductor physics}

Silicon is the most frequently used semiconducting material for biointerface studies. Other semiconductor materials such as $\mathrm{CdSe} / \mathrm{ZnS}$ nanoparticles ${ }^{17}$ and $\mathrm{ZnO}$ microwires ${ }^{34}$ have also been explored owing to their advantageous properties, including well-defined photoluminescence ${ }^{17}$ and optical transparency ${ }^{34}$, for applications such as fluorescence 
imaging ${ }^{18}$, integrated light delivery and electrical recording ${ }^{34}$. Important materials considerations for building a bioelectronic or biophotonic interface include carrier mobility and doping types (that is, p-type, n-type or intrinsic), whether a semiconductor has a direct or indirect bandgap (FIG. 2a) and the position of the Fermi levels and band edges with respect to the redox potential of the chemical species in biological fluids (FIG. 2b).

\section{Carrier mobility and doping}

Carrier mobility characterizes how fast a charge carrier (that is, an electron or a hole in a semiconductor) moves through a material in an electric field ${ }^{7,8}$. The electrical conductivity of semiconductors can be modified by the introduction of electronic impurities into the crystal lattice, which is called doping ${ }^{7,8}$. At biointerfaces, the carrier mobility and doping type of a semiconductor component in a FET determine the sensitivity or gain, that is, the transconductance $\left(g_{\mathrm{m}}\right)$. The transconductance is the ratio of the change in drain-source current $\left(I_{\mathrm{DS}}\right)$ to the change in gate-source voltage ( $V_{\mathrm{GS}}$ ) over a defined interval on the $I_{\mathrm{DS}}-$ $V_{\mathrm{GS}}$ curve. Moreover, the doping profile determines whether a cathodic or anodic reaction takes place during the photoelectrochemical modulation of tissues (FIG. 2c).

\section{Bandgap}

The type of bandgap — direct or indirect — affects the intrinsic light absorption coefficient and thus light emission through optical or electrical excitation ${ }^{7,8}$. Illumination of a direct bandgap semiconductor generates excited electrons in the conduction band and holes in the valence band. Recombination of these carriers can be radiative if the conduction band minimum and valence band maximum are aligned in the momentum space, giving rise to photoluminescence (FIG. 2a). In an indirect bandgap semiconductor, phonons (that is, lattice vibrations) are involved in light absorption and emission (FIG. 2a). Phonons allow a semiconductor to convert the energy of light into either thermal (that is, photothermal) ${ }^{35}$ or mechanical (that is, photoacoustic) $)^{36}$ responses, which can affect an interfacing biological component. Therefore, the band structure of a semiconductor determines the effect or the design of a device at the biointerface. For example, direct bandgap semiconductors allow for light-emitting diode (LED)-based optogenetic control ${ }^{24}$ and photoluminescence-enabled subcellular tracking ${ }^{37}$, whereas indirect bandgap semiconductors can be used for the photothermal modulation of neurons ${ }^{29}$ or photoelectrochemical biofuel production after the incorporation of a light-trapping structure to improve light absorption ${ }^{28}$.

\section{Fermi levels}

The position of the Fermi level $\left(E_{\mathrm{F}}\right)$ in relation to the band energy levels is an important factor in determining the electrical properties of a semiconductor. Fermi levels and both conduction band and valence band edge positions for dry-state devices are usually plotted with respect to the vacuum level. However, to consider the charge transfer of the solution species at a biointerface, the normal hydrogen electrode (NHE) or standard hydrogen electrode (SHE) potential constitutes a more adequate reference ${ }^{38}$ (FIG. 2b), because the redox potentials of the specific solution species are considered (for example, $\mathrm{H}_{2} \mathrm{O} / \mathrm{H}_{2}$ at a $\mathrm{pH}$ of $~ 7.4)$. This consideration is particularly important when comparing semiconductors with different band edge positions for biophysical studies (FIG. 2b). Moreover, the relative positions of semiconductor Fermi levels with respect to solution redox potentials determine 
the electrochemical or photoelectrochemical reactions occurring at the semiconductor surface (FIG. 2c). The Faradaic reactions at the biointerface and the coupling reactions (that is, the anodic reaction is the coupling reaction to the cathodic reaction) that are spatially separated from the biointerface can shift the local electrochemical potential and, correspondingly, the cell membrane voltage (that is, the difference between intracellular and extracellular potentials) and modify the local chemical environment, affecting cellular physiology and potentially making the semiconductor cytotoxic.

\section{Other important properties}

In addition to these three fundamental features, band bending at the semiconductor-biofluid interface ${ }^{39}$, semiconductor surface charges and potentials ${ }^{38}$, light-induced carrier dynamics, interfacial charge transfer and surface states ${ }^{38}$ are important semiconductor properties. For example, if a semiconductor is immersed in a saline solution containing ions and redox species (FIG. 2c), the charge flow between the solid and liquid phases aims to reach an equilibrium in terms of the Fermi levels $\left(\mathrm{E}_{F}=E_{\mathrm{F}}(\text { redox })\right)^{38,40}$. This spontaneous process typically results in band bending at the surface of the semiconductor phase ${ }^{39}$ (FIG. 2c). In an n-type semiconductor, the donor impurities provide the majority carriers (that is, electrons) required for Fermi level equilibration (FIG. 2c). Upon charge transfer, the remaining semiconductor region contains positive charges from the ionized donors, which produce a space charge region ${ }^{39}$. The counter charge is located on the electrolyte side, which is very close to the interface and within the Helmholtz double layer. The size of the space charge region depends on the doping level, with a lightly doped semiconductor resulting in a larger space charge width ${ }^{39}$. Photocarriers generated by illumination of a semiconductor in a biological fluid generate quasi-Fermi levels for both electrons and holes $\left(E_{\mathrm{F}}(\mathrm{e})\right.$ and $E_{\mathrm{F}}(\mathrm{h})$, respectively). The space charge region drives photoelectrochemical reactions for biomolecular sensing or cellular stimulation if $E_{\mathrm{F}}($ redox $)$ is correctly aligned with $E_{\mathrm{F}}(\mathrm{e})$ or $E_{F}(h)^{38}$ (FIG. 2c). Finally, the surface states of semiconductors play an important role in the design of biointerfaces. Surface states usually occur as a result of the abrupt termination of the lattice structures at the solid-liquid interface or because of surface impurities, such as metal atoms or absorbed solution species ${ }^{38}$. These surface states can exchange charges with the conduction and valence bands of the semiconductor and those of the redox species in biological fluids.

\section{Operation at biointerfaces}

A FET device consists of a semiconductor channel, a dielectric layer and three electrodes (source, drain and gate) (FIG. 3a). A small shift in the $V_{\mathrm{GS}}$ results in a large change in the $I_{\mathrm{DS}}$. In bioelectronic sensing, $V_{\mathrm{GS}}$ is caused by a local electrochemical potential or charged species in saline. FET-based devices can detect local potentials originating from dynamic ion flows, static ion gradients and device surface charges.

Dynamic ion flows, which can, for example, be initiated by tissue damage, produce endogenous bioelectric fields in the extracellular space ${ }^{41}$, as defined by Poissons equation ${ }^{42}$ : 


$$
\varphi\left(x^{\prime}, y^{\prime}, z^{\prime}\right)=\frac{1}{4 \pi \sigma} \int \frac{I_{v}(x, y, z)}{r\left(x, y, z, x^{\prime}, y^{\prime}, z^{\prime}\right)} \mathrm{d} x \mathrm{~d} y \mathrm{~d} z
$$

where $\varphi\left(x^{\prime}, y^{\prime}, z^{\prime}\right)$ is the potential at $\left(x^{\prime}, y^{\prime}, z^{\prime}\right)$, a is the electrical conductivity of the medium, $I_{V}$ is the volumetric source current density at position $(x, y, z)$ and $r(x, y, z, x, y$, $\left.z^{\prime}\right)$ is the distance between $(x, y, z)$ and $\left(x^{\prime}, y^{\prime}, z^{\prime}\right)$. Poisson's equation can be used to qualitatively assess extracellular electronic sensing. For example, to increase $\varphi, \sigma$ can be decreased by limiting ion mobility via reduction of the distance between the FET device and the cell membrane by means of either mechanical pressing or substrate topography engineering $31,43-45$.

Static ion gradients can establish resting transmembrane potentials for cells and organelles, as described by the Goldman-Hodgkin-Katz equation:

$$
V_{m}=\frac{R T}{F} \ln \frac{\Sigma_{i}^{N} P_{M_{i}^{+}}\left[M_{i}^{+}\right]_{\text {out }}+\Sigma_{j}^{M} P_{A_{j}^{-}}\left[A_{j}^{-}\right]_{\text {in }}}{\Sigma_{i}^{N} P_{M_{i}^{+}}\left[M_{i}^{+}\right]_{\text {in }}+\Sigma_{j}^{M} P_{A_{j}^{-}}\left[A_{j}^{-}\right]_{\text {out }}}
$$

where $V_{m}$ is the membrane potential, $R$ is the ideal gas constant, $T$ is the absolute temperature, $F$ is the Faraday constant and $P_{M_{+}}$and $\left[M^{+}\right]$represent the permeability and concentration of a monovalent $M^{+}$ion, respectively.

A change in surface charges can be caused by the binding of a charged biomolecule or a particle, for example, an influenza type A virus ${ }^{46}$, or by exposure to biological fluids with a different $\mathrm{pH}$, which can, for example, be caused by a local inflammatory response $\mathrm{e}^{22}$. The effect of surface charges on the FET response is relevant to the ion concentrations and is limited by a characteristic length described by Debye screening:

$$
\lambda_{\mathrm{D}}=\frac{1}{\sqrt{4 \pi l_{\mathrm{B}} \Sigma_{i} \rho_{i} z_{i}^{2}}}
$$

where $I_{\mathrm{B}}$ is the Bjerrum length, $\Sigma_{i}$ is the sum over all ion species and $\rho_{i}$ and $z_{i}$ are the concentration and valence of ion species $i$, respectively. The Debye screening length defines 
an approximate limit above which the response of a FET device substantially decreases (owing to the weakened electrostatic effect in saline). $\lambda_{\mathrm{D}}$ of a $1 \times$ phosphate buffered saline is $\sim 0.7 \mathrm{~nm}\left(\mathrm{REF}^{47}\right)$. To overcome this limitation, saline solutions with low ionic strength can be used for biomolecular sensing. Alternatively, sophisticated FET surface modifications, for example, with deoxyribonucleotide aptamers ${ }^{48}$, or specialized device operations ${ }^{49}$ can be explored.

For a FET based on an n-type semiconductor, a positive gate voltage bias ( $V_{\mathrm{GS}}>0$, for example, through an increase in the local electrochemical potential) or binding of a positively charged species causes the accumulation of majority carriers (electrons) at the semiconductor-dielectric interface and a corresponding increase in $I_{\text {DS }}$ (FIG. 3a). The sensitivity of the $I_{\mathrm{DS}}$ change is defined as the transconductance $\mathrm{g}_{m}$, which is a key characteristic of a FET sensor. In the linear regime, $g_{\mathrm{m}}$ typically follows $g_{\mathrm{m}} \propto \mu V_{\mathrm{DS}}$, where $\mu$ is the charge carrier mobility of the semiconductor and $V_{\mathrm{DS}}$ is the drain-source voltage. The transconductance $g_{\mathrm{m}}$ can be used to convert the recorded FET conductance or current change into corresponding electrochemical potential changes.

Therefore, FET devices can be used for extracellular recordings from tissues and organs, intracellular recordings from the cytosol or organelles, and biomolecular or chemical sensing, for example, to detect stochastic molecular binding events ${ }^{50}$, conformational changes in charged molecular backbones ${ }^{48}$ or analyte concentration from a blood sample ${ }^{51}$.

A p-n or a p-i-n diode junction (FIG. 3b) is the key building block for optoelectronic devices, such as photo-voltaic devices (FIG. 3c) and LEDs (FIG. 3d). If p-type and n-type semiconductors are in contact, their valence band and conduction band edge positions shift to account for the energy difference between their original Fermi levels. At the equilibrium state, a built-in electric field is established across the diode junction to balance the charge carrier flow. In a photovoltaic device (FIG. 3c), incident illumination can create electronhole pairs in the bulk, splitting the dark Fermi levels into quasi-Fermi levels for electrons $E_{\mathrm{F}}(\mathrm{e})$ and holes $E_{\mathrm{F}}(\mathrm{h})$, with an energy difference of $\mathrm{eV}_{p h}$. The minority carriers diffuse to the space charge region and are swept out by the built-in electric field, producing a photocurrent $I_{\text {ph}}$. Thus, a photovoltaic device can either serve as a power source for electrodes to stimulate cells or tissues or directly establish biointerfaces for light-enabled capacitive or Faradaic stimulation. In an LED device (FIG. 3d), a forward bias $V_{\text {bias }}$ is applied to the diode junction (the potential at the p-terminal is higher) to overcome the barrier of the built-in potential, and the majority carriers flow across the junction. Recombination near the junction and within the carrier diffusion length in the bulk semiconductor leads to the emission of photons in the case of a direct band-to-band transition. LED devices are typically used as light sources for optogenetic applications.

\section{Key advantages}

Inorganic semiconductors operate via mechanisms different from those of metal-based or polymer-based devices and typically comprise active device components. For example, semiconductor bioelectronic sensors usually use a FET configuration, as opposed to an electrochemical device incorporated in metal-based sensors. Inorganic semiconductor 
systems (for example, FETs, photovoltaic devices or LEDs) still unimpededly function and can even acquire new functions if the size of the device is reduced to nanometres ${ }^{52-56}$, compared with metal-based or polymer-based devices (FIG. 3). Being functional at the nanoscale not only improves the precision and accuracy of current measurements but also enables subcellular studies (for example, probing the electrodiffusion at a synaptic junction $^{57}$ ).

Inorganic semiconductor devices can be processed in a highly scalable manner ${ }^{58,59}$ owing to the availability of well-established technologies for the fabrication of complementary metaloxide-semiconductor (CMOS) integrated circuits. CMOS integrated circuit technology enables the design of multi-electrode arrays for the mapping of electrophysiology in a cellular network ${ }^{60-65}$. For example, mechanically flexible Si-based sensor systems, composed of 2,016 Si nanomembrane transistors, can be used to directly record the electrical activities from a beating porcine heart in vivo with high spatiotemporal resolution ${ }^{63}$. The high sensor density of the device allows the spatial mapping of spontaneous and paced ventricular depolarization along the epicardial surface ${ }^{63}$. Similarly, the Neuropixels $\mathrm{Si}$ probes contain 960 CMOS processing-compatible recording sites to capture well-isolated spiking activities from hundreds of neurons in freely moving animals ${ }^{65}$. Notably, the combination of high-performance electrode technology and scalable semiconductor chip fabrication enables the measurement of brain-wide neural activity at cellular resolution and subcellular resolution during complex behaviours ${ }^{65}$.

Semiconductor devices can further efficiently harvest radiant energy and convert it into other forms of energy, such as electricity ${ }^{40-56}$, making the operation of miniaturized wireless devices possible ${ }^{30-66-67}$. For example, most electronic retinal prostheses are powered by inductive coils, the implantation of which requires a complex surgical procedure. In a photovoltaic subretinal prosthesis, the miniaturized silicon photodiodes at each pixel directly receive the light signal and the power to electrically stimulate neurons ${ }^{67}$.

Inorganic semiconductor devices and circuits are characterized by fast response, signal amplification, multi-functionality and multiplexity and are faulttolerant ${ }^{68}$, making them ideal for assessing complex dynamics in biological systems. For example, inorganic semiconductors could be used for in situ biochemical and biophysical measurements to detect molecular and cellular heterogeneities or transient biological interactions and to analyse network-level feedback, which are difficult to assess by other tools, such as the patch clamp technique or atomic force microscopy. Finally, interesting semiconductor properties, such as the flexo-photovoltaic effect ${ }^{69,70}$ or intrinsic ductility ${ }^{71}$, can potentially be leveraged for biointerface studies in the future.

\section{Semiconductor design parameters}

\section{Quantitative parameters}

Important quantitative considerations for semiconductor-based interfaces include the length and time scales of measurements, electrical and mechanical properties of the semiconducting material, and energy input and output (TABLE 1). The properties, energy and scales of the semiconductor and the biological components need to be considered for the 
design of the biointerface. For example, single-crystalline Si nanowires with diameters of $\sim 100 \mathrm{~nm}$ can be stretched up to $\sim 16 \%$ tensile strain at room temperature ${ }^{72}$, approaching the theoretical elastic limit of $\mathrm{Si}(17-20 \%)$ (TABLE 1). Therefore, the elastic behaviour of Sinanowire-based nano-electronics enables interfacing with soft and deformable biological components ${ }^{1}$ such as heart tissue. Photoelectrochemical cells can also be potentially applied for wound healing; for example, the photocurrent density of a photoelectrochemical cell (current density of $\sim 35 \mathrm{~mA} \mathrm{~cm}^{-2}$ ) 126 is large enough to alter the endogenous bioelectric field near a cornea wound (current density of $\left.\sim 5 \mu \mathrm{A} \mathrm{cm}^{-2}\right)^{41}$.

Many biointerfaces further involve energy transduction and thus the balance of energy input and output can be used to predict the experimental outcome (TABLE 1). For example, for a 2- $\mu \mathrm{m}$-long coaxial Si nanowire with a diameter of $300 \mathrm{~nm}$, the photovoltaic power output under one sun illumination is on the order of $\sim 10^{-10}-10^{-9} \mathrm{~W}$ ( $\mathrm{REF}^{56}$ ), which is two orders of magnitude larger than the power consumption of a similarly sized bacterial cell $\left(\sim 10^{-12}\right.$ $\mathrm{W})^{73}$. Therefore, single Si nanowires can be used to modulate the physiology of individual bacterial cells using sunlight ${ }^{28}$.

The length scale is another critical parameter for the design of biointerfaces. Although various energy terms (for example, chemical, mechanical, thermal and electrostatic energy) depend on the size of both the device and the biological components at the biointerface ${ }^{74}$, their amplitudes converge at the molecular and subcellular scales. At these scales, the differences between hard and soft materials and between living and non-living systems become diminished (TABLE 1). For example, by reducing the thickness of a Si membrane to $10 \mathrm{~nm}$, it can achieve a tissue-level bending stiffness $\left(10^{-5} \mathrm{nN}\right.$ m for a 10-nm-thick Si and $10^{-4} \mathrm{nN} \mathrm{m}$ for a $20-\mu \mathrm{m}$-thick brain slice ${ }^{75}$ ), providing a mechanically matched bioelectronic or biophotonic interface. Nevertheless, the precise control of biological activities through physical stimulation at the molecular and subcellular scales remains a challenge.

Understanding the impact of the size of the biointerface on biological processes will enable the design of the correct material dimensions, device geometry and physical properties to improve integration, signal transduction and biocompatibility.

\section{Semiconductor materials}

The chemistry, structure, stability and control of semiconductor materials are important for the design of biointerfaces. For example, biodegradable semiconductors are required for certain biointerface studies, such as for transient biological sensing ${ }^{16}$ or modulation ${ }^{30}$, while other applications, such as chronic implantable devices, need stable materials ${ }^{76}$.

Geometry.-Semiconductors with different geometries, that is, $0 \mathrm{D}^{37,77}, 1 \mathrm{D}^{1,78-80}, 2 \mathrm{D}^{81-84}$ and $3 \mathrm{D}^{3,29,85,86}$, have been designed for establishing functional biointerfaces (FIG. 4a-d), for example, for subcellular transport studies ${ }^{30,37}$, intracellular FET-based electrophysiology ${ }^{31}$, light-driven excitation of neural activities ${ }^{29,30,67,87-90}$ and electronicsintegrated engineered tissues ${ }^{22,91,92}$. The choice of semiconductor geometry depends on the biological target, for example, an organelle or a patch of skin surface, or the biological problem, for example, intracellular or extracellular recording. Independent of the geometry, the size of the semiconductor can drastically change the mechanical properties of the 
material, which is key to establishing certain biointerfaces, such as epidermal electronics ${ }^{93}$. For example, the bending stiffness of a 2D semiconductor sheet is defined as $D=E h^{3} / 12(1-$ $v^{2}$ ), where $D$ is the bending stiffness, $E$ is the Young's modulus, $h$ is the thickness and $v$ is the Poisson ratio ${ }^{81,94}$, and therefore, the thickness of the sheet determines whether an epidermal device can provide a conformai interface on $\operatorname{skin}^{93}$.

Chemical synthesis.- Solution-phase colloidal synthesis and vapour-phase conversion have long been used for growing semiconductor materials, with many parameters being readily programmable, including the growth temperature and substrate orientation. In particular, chemical vapour deposition can be used for the scalable production of various semiconductor nanostructures, such as uniform and heterostructured nanowires ${ }^{1,31,95,96}$, multilayered membranes ${ }^{30}$ and mesostructured particles ${ }^{29}$. One noteworthy and underappreciated method is the electrochemical synthesis of specialized semiconductors ${ }^{15,97-99}$. For example, porous Si films can be produced by electrochemical etching of single-crystal Si wafers in ethanolic hydrofluoric acid solution. These films can be lifted off the etching substrate, followed by ultrasonication and filtration, to achieve a narrow particle size distribution. In addition to electrochemical etching, other chemical etching techniques, such as metal-assisted etching and stain etching, can be applied to fabricate a variety of porous Si morphologies, including wires ${ }^{100}$, cones $^{101}$ and lamellae ${ }^{102}$.

Chemical processes can control not only the structure and chemical composition of semiconductors but also the physical properties that are highly relevant to biointerfaces and biophysical studies $29,30,98,99,103$. For example, the porous structures in $\mathrm{Si}$, introduced by nanoscale casting or electrochemical etching, typically cause a substantial reduction in the effective Young's modulus compared with the bulk material ${ }^{29,101,104}$. This change in material mechanics through chemical processes allows a material to establish minimally invasive contact with cells and tissues ${ }^{29,101}$. Similarly, electrochemically etched Si can be treated in an aqueous solution to tailor its photoluminescence ${ }^{98,105,106}$. Surface states can be generated in porous $\mathrm{Si}$ if the hydrogen-terminated $\mathrm{Si}$ surface is oxidized in water. These states and a possible quantum-confinement effect likely contribute to the spectral features and quenching kinetics of the photoluminescence ${ }^{98}$.

Moreover, various semiconductor-specific properties can be tuned by specific material synthesis techniques ${ }^{107}$, including the bandgap, which affects light absorption and conductivity, the carrier mobility $(\mu)$ and lifetime $(\tau)$, which define the carrier diffusion length $\left(L_{\mathrm{D}}\right)$ by $L_{\mathrm{D}}=\sqrt{k_{\mathrm{B}} T \mu \tau / \mathrm{e}}$, and the surface states, which tune the band edge position with respect to a target redox potential. For example, bulk molybdenum disulfide $\left(\mathrm{MoS}_{2}\right)$ is an indirect bandgap semiconductor with a gap size of $1.3 \mathrm{eV}\left(\mathrm{REF}^{108}\right)$. Chemically synthesized $\mathrm{MoS}_{2}$ (for example, vertically aligned nanofilms ${ }^{83}$ ) can have a substantially reduced total layer thickness and thus produce a direct bandgap with an increased gap size (up to $1.9 \mathrm{eV}$ ). This feature has been exploited for nanostructured $\mathrm{MoS}_{2}$-based retinal prosthetics $^{82}$ and disinfection ${ }^{83}$ applications.

Surface functionalization can further provide an additional layer of control in biointerface studies. Surface conjugation with ligands, antibodies or peptides improves cellular targeting efficiency ${ }^{109,110}$. For example, the surface of Si nanowires can be functionalized with the 
cell-penetrating peptide transactivating transcriptional activator from $\mathrm{HIV}-1$ to facilitate spontaneous internalization into primary neuronal cells ${ }^{111}$. Similarly, kinked Si nanowire FETs comprising probe tips that are coated with phospholipid bilayers enable a minimally invasive entrance into cells for the high-quality recording of intracellular potentials ${ }^{31}$. Contrarily, undesired protein absorption on semiconductor surfaces can also lead to deleterious performance, which has to be taken into account in the design of semiconductor materials ${ }^{112-114}$.

Stability in physiological conditions.-The stability of semiconductors in physiological conditions is an important consideration for biointerface design. Si is commonly used in electronics and optoelectronics owing to its well-studied and stable physical properties in the dry state and established fabrication technologies. The biodegradability of $\mathrm{Si}$ is of particular interest for wet applications, for example, in transient electronics $^{115}$, for in vivo photoluminescence $98,101,105$ and for sustained molecule release ${ }^{101,104,116}$. The degradation of Si in biological fluid depends on its porosity, its dopant type and concentration, its crystal quality, the $\mathrm{pH}$ of the fluid, its ionic strength and the temperature ${ }^{15,117-120}$. For example, $1 \mu \mathrm{m}$-thick mesoporous Si with high porosity (70\%) and a high surface area $\left(\sim 640 \mathrm{~m}^{2} \mathrm{~g}^{-1}\right)$ completely degrades in saline within a day ${ }^{15}$.

Si-based materials and devices can stably perform in the wet state if their surfaces are passivated ${ }^{121-126}$. For example, to improve the stability of Si photoelectrodes, a thin epitaxial layer of strontium titanate $\left(\mathrm{SrTiO}_{3}\right)$ can be grown directly on $\mathrm{Si}(001)$ by molecular beam epitaxy ${ }^{126}$. Despite passivation, photogenerated electrons are easily transported through the epitaxial layer owing to the conduction band alignment and lattice match between single-crystalline $\mathrm{SrTiO}_{3}$ and $\mathrm{Si}\left(\mathrm{REF}^{126}\right)$. This composite can be used as an efficient and stable photocathode for water splitting ${ }^{126}$ and could potentially be applied for biointerface studies. Leakage-free and biocompatible thermal oxide, synthesized directly from Si wafers, can inhibit Si degradation in saline ${ }^{121}$. The ultrathin thermal oxide layer completely seals the underlying electronics, enabling capacitive coupling with tissues ${ }^{121}$.

Therefore, the lifetime of semiconductors can be controlled by their bulk and surface properties $^{121-126}$, including dimensions, doping profiles, crystallinity, porosity and surface passivation. By modifying these properties, Si-based devices can be designed for temporary or continuous operation and tailored for specific applications (for example, drug delivery requires transience, and chronic implants require stability). In addition to material considerations, extrinsic parameters, such as saline temperature, ion type and concentration, and the biological targets, can also substantially affect the degradation kinetics of semiconductors $115,117,118$.

\section{Biointerfaces}

The semiconductor-cell interface can be either an extracellular or an intracellular biointerface (FIGS 4e, 5a; TABLE 2). In particular, nanoscale semiconductors can enter a cell and thus can form intracellular biointerfaces for either sensing or stimulation $^{30,31,111,127}$. A clear understanding of semiconductor internalization mechanisms remains elusive thus far. However, substrate-bound device internalization is typically 
achieved by electroporation ${ }^{86,128}$, optoporation ${ }^{129}$ or surface modification ${ }^{31,111}$.

Freestanding materials or devices can be internalized through spontaneous

endocytosis ${ }^{127,130,131}$ or microinjection ${ }^{132}$. Intracellular semiconductors are in direct contact with organelles and/or the cytosol, enabling high-fidelity studies of subcellular activities $^{30,133}$. Biointerfaces can be categorized on the basis of the function of the semiconductors (FIG. 4e).

\section{Electronic sensing}

Semiconductors can perform electronic sensing by converting a biological signal, represented by ions, into a current and/or voltage (TABLE 2). FETs (FIG. 3a) can be used to sense $\mathrm{pH}^{22,31,134}$, the concentration of proteins, nucleic acids ${ }^{134,135}$ and viruses ${ }^{46}$, and bioelectric signals (for example, action potentials) emitted by cells or tissues $1,91,136$. Compared with metal-based devices or organic FETs, inorganic FETs can operate at a larger switching speed (for example, up to $2 \mathrm{THz}$ for core-shell Ge/Si nanowires ${ }^{137}$ ), which allows them to electrically resolve most biomolecular and cellular activities ${ }^{73}$. In particular, $\mathrm{Si}$ nanowires ${ }^{1,25,78,91,138}$, which constitute a key building block of nano-bioelectronics ${ }^{1}$, are very sensitive to changes in electrical potentials and surface charges and are more stretchable and bendable than bulk materials ${ }^{72}$.

A striking feature of nanowire-based electronic devices is their ability to record electrical signals from a localized region of a cell population, such as an intracellular space $^{31,60,86,128,133,139-141}$. In contrast to extracellular electrical potential or charges, intracellular signals are more difficult to measure because the electrical probe has to be inserted into a cell, which constitutes an invasive step and thus might alter cellular signalling. To provide a more deformable and therefore less-interfering probe, a flexible nanoscale FET device can be fabricated by integrating a multiply kinked Si nanowire into a metal-polymer-based support ${ }^{31,133}$. The kinked nanowire tip has a $60^{\circ}$ junction angle, which facilitates easy insertion of the device tip into a cell, for example, into a cultured cardiomyocyte ${ }^{31}$. Additionally, the probe can be coated with a phospholipid bilayer to promote cellular penetration through membrane fusion. This point-like FET can measure the intracellular potential adjacent to the plasma membrane and minimizes chemical and mechanical perturbation of the cells.

A different FET sensing configuration can be achieved by remote capacitive coupling; bioelectric signals from cells and tissues can be capacitively coupled to the gate electrodes of a FET device. Such a device configuration requires a complex device design; however, all key FET components (that is, source, drain, gate and gate dielectrics) can be isolated from the biological fluid, which substantially improves device longevity in vivo ${ }^{121}$.

An important engineering consideration is the relative position of the FET device and the signal source. For extracellular electric potential sensing, the distance between the plasma membrane of the cell and the FET surface (that is, the biointerface cleft) substantially affects the junction voltage $\left(V_{\mathrm{j}}\right)$ (FIG. 5a) and the recorded signal amplitude (that is, from the device noise level up to a few $\mathrm{mV}$ ). The cleft in the planar biointerface is typically greater than $\sim 40 \mathrm{~nm}$ (REFS ${ }^{142,143}$ ), but it can be reduced to improve the FET recording signal by 
mechanical pressing or topography engineering of the device substrate ${ }^{43,45}$. Such distance effect is not applicable to intracellular potential sensing.

\section{Optoelectronic light sensing}

Semiconductors can also respond to light, which can be translated into a current and/or voltage signal (TABLE 2). Light can originate from biological components as bioluminescence ${ }^{144}$ or as the fluorescence of an intracellular calcium indicator. Light emission can be sensed by a semiconductor device (FIG. 5a), usually through a photo-detector that contains $\mathrm{p}-\mathrm{i}-\mathrm{n}$ or $\mathrm{p}-\mathrm{n}$ diode junctions $\mathrm{s}^{7,8}$ (FIG. 3b). For example, the integration of illumination LEDs, CMOS image sensors, wireless power and data transmission circuits into a single chip enables wireless capsule endoscopy ${ }^{145}$. Alternatively, an ingestible microbioelectronic device can be used to directly and selectively sense biomolecules in vivo ${ }^{144}$. In this device, engineered bacteria transduce blood signals into bioluminescence, which is detected by a semiconductor-based detector and wirelessly transmitted to an external receiver. Similarly, a polymer-supported, wireless, injectable fluorescence photometer, which includes a photodetector based on $\mathrm{GaAs} / \mathrm{In}_{X} \mathrm{Ga}_{1-X} \mathrm{P} / \mathrm{Al}_{y} \mathrm{Ga}_{1-y} \mathrm{As}$, can record calcium fluorescence in the deep brain of untethered and freely moving animals, who are genetically encoded with calcium indicators ${ }^{146}$.

\section{Optoelectronic stimulation}

Photovoltaic, photocapacitive, photoelectrochemical and photoconductive stimulation.-For optoelectronic stimulation, a light signal is converted by the semiconductor into electrical or electrochemical outputs to modulate the concentration distribution of ions or the redox state of molecules; alternatively, light is converted in a cell to elicit a biological signal (BOX 1; TABLE 2). A photovoltaic device is typically a dry-state component that contains a diode junction ${ }^{40}$, which also provides all the voltage ( $V_{\mathrm{S}}$ ) (FIG. 5a) needed for the stimulation electrodes ${ }^{66,67,88,89,147}$ (that is, metals or conducting metal oxides). Alternatively, a photovoltaic material can be directly exposed to saline for either capacitive or Faradaic stimulation (FIG. 5b), depending on the nature of the semiconductorsaline interface. A photocapacitive effect is typically transient and caused by the redistribution of ions near the double layer, whereas a photoelectrochemical process typically involves a redox reaction with an interfacial Faradic impedance $\left(Z_{S}\right)$ (FIG. 5a), which is long-lasting during illumination. Photocapacitive $e^{30,148-150}$ or photoelectrochemical ${ }^{27,28,30,151,152}$ processes have primarily been used in extracellular stimulation to modulate ion redistribution or changes in chemical species at plasma membranes. A photoconductive process refers to a light-induced increase in charge injection at the device-liquid interface, with an already electrically biased device (that is, external power is still needed). This process typically involves reactions at the semiconductorsaline $^{87}$ or electrode-saline ${ }^{66,67,82}$ interface.

Si-based photovoltaic devices without an external power source can be used to stimulate retinal tissues in vivo with high-intensity, pulsed near-infrared (NIR) light $66,67,88,89,147$. For example, sputtered iridium oxide film (SIROF) electrodes, coupled with multiple Si diodes, can be connected in series to sense NIR light and to simultaneously produce the electrical power driving electrochemical charge injection at the SIROF surface. Alternatively, 
optoelectronic retinal prosthetics can be made of a curved heterostructure consisting of $\mathrm{MoS}_{2}$ and graphene (photo-absorbing layer and interconnect) ${ }^{82}$. Local light-induced conductance changes in the $\mathrm{MoS}_{2}$ /graphene structure are amplified and converted by external flexible circuits into programmed electrical stimuli to the retina.

Most optoelectronic stimulation devices require interconnects or internal wiring; however, freestanding Si membranes containing $\mathrm{p}-\mathrm{i}-\mathrm{n}$ diode junctions can be used for the photocapacitive stimulation of neurons, glia and other mammalian cells ${ }^{30}$. By coating the surfaces of the diode junctions with metal nanoparticles, both capacitive and Faradaic (FIG. $5 b)$ currents are substantially enhanced, resulting in efficient wireless stimulation of the brain cortex and behaviour control in a mouse model ${ }^{30}$. The semiconductor structures and mechanics have to match those of the biological targets to establish tight junctions. The desired photoresponses can then be tuned by modulating the Si doping profile, surface chemistry and dimensions to promote specific light-triggered effects ${ }^{30}$. Similarly, atomic, metal-covered $\mathrm{p}-\mathrm{i}-\mathrm{n}$ coaxial Si nanowires can be used for the photoelectrochemical modulation of single neuronal activities ${ }^{151}$.

Semiconductor nanomaterials without diode junctions or other heterogeneous structures can also be used for the photoelectrochemical modulation of enzymatic or cellular activities $^{27,28,153,154}$ (FIG. 5a). For example, cadmium sulfide (CdS) nanocrystals can be used to photosensitize the nitrogenase molybdenum-iron $(\mathrm{MoFe})$ protein ${ }^{151}$; in this case, light harvesting (instead of ATP hydrolysis) drives the enzymatic reduction of $\mathrm{N}_{2}$ into $\mathrm{NH}_{3}$. CdS nano-particles can also be precipitated from bacterium cell walls for the selfphotosensitization of the non photosynthetic bacterium Moorella thermoacetica ${ }^{11}$ (FIG. ). This biohybrid can photosynthesize acetic acid from carbon dioxide and thus offers a selfreplicating device for solar-to-chemical carbon dioxide reduction. Acetogens, which are microorganisms that generate acetate, for example, Sporomusa ovata, can also form hybrids with silicon nanowires for the light-driven production of acetate 28,155 (FIG. 4b). Light excitation generates electrons from silicon, providing an energy source for $\mathrm{CO}_{2}-$ the carbon source of bacteria. The acetate can be coupled to Escherichia coli production strains to produce other valuable products such as acetyl coenzyme A and $n$-butanol ${ }^{28,155}$. Under aerobic conditions, $\mathrm{O}_{2}$ itself can serve as an electron acceptor and can be converted into reactive oxygen species (ROS), such as a hydroxyl radical, singlet oxygen or superoxide. ROS generated from semiconductors (for example, at the edges of vertically stacked $\mathrm{MoS}_{2}$ nanostructures ${ }^{83}$ ) are strong oxidants that can inhibit bacterial growth and even achieve complete disinfection ${ }^{83,156}$. ROS can also accumulate during the photoelectrochemical stimulation of mammalian cells, causing cell damage or death ${ }^{157}$.

These photoelectrochemical reactions involve electrons, stimulating saline-mediated biological reactions; the electrons have to be coupled with hole reactions if charging of semiconductors is not considered. Potential hole scavengers under biological conditions

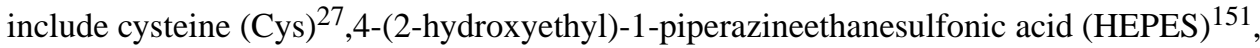
3-( $N$-morpholino)propanesulfonic acid (MOPS $)^{151}$, 2-morpholin-4-yl-ethanesulfonic acid $(\mathrm{MED})^{151}$, Tris ${ }^{151}$ and ascorbic acid ${ }^{151}$. 
LED-based stimulation.-LEDs can also be used for the stimulation of biological signals. Applying a current or voltage to an LED causes the emission of light, triggering a biological response (TABLE 2). Although the interface between an LED and a biological system is remote (FIG. 5a), LEDs are very important tools for photostimulation applications, including optogenetics and non-genetic techniques, such as the use of metals or semiconductors for the converting of light signals into biologically relevant forms (for example, electrical, thermal and mechanical signals). Microfabricated LEDs can be integrated with flexible and bioresorbable substrates for in vivo applications, for example, for brain ${ }^{146,158}$ or spinal cord ${ }^{90,158}$ stimulation. Importantly, voltage can be wirelessly applied to LEDs by either radio frequency radiation ${ }^{24}$ or infrared light ${ }^{159}$, enabling a compact and less invasive biointerface without the need for supporting equipment. For example, upon infrared light illumination, two or more GaAs photodetectors that are connected in series can produce sufficient photovoltage to run a visible LED, which emits red (with $\mathrm{Ga}_{0.5} \mathrm{In}_{0.5} \mathrm{P}$ ) or yellow (with $\mathrm{Al}_{0.15} \mathrm{Ga}_{0.35} \mathrm{In}_{0.5} \mathrm{P}$ ) light for optogenetic stimulation in vivo ${ }^{159}$; this setup provides a device for infrared-to-visible upconversion.

\section{Photothermal stimulation}

For photothermal stimulation, a light pulse is absorbed by the semiconductor and converted into transient heat to elicit a biological response (TABLE 2). In contrast to the photovoltaic effect, the photothermal effect receives less attention but can have a profound effect on the photostimulation of biological systems. Upon optical excitation of a semiconductor, the input photon energy is dissipated through a non-radiative recombination process involving phonons ${ }^{35}$. Corresponding lattice vibrations induce a local temperature increase, which can be harnessed to trigger a biological response. In the photoresponse of Si structures, the relative output amplitudes of thermal versus electrical signals can be tuned by varying the dimensions of the materials, the doping and surface chemistry and the illumination intensities ${ }^{30}$. For example, a predominant photothermal effect (that is, light-induced electrical signals from the semiconductors are negligible) can be achieved by delivering a focused laser light to nanocrystalline intrinsic Si nano-wires ${ }^{30}$ or mesoporous Si particles with an amorphous framework ${ }^{29}$.

Semiconductor-based photothermal interfaces (FIG. 5a) with cells and organelles can be established in vitro ${ }^{29,30}$. Compared with metals or carbon, which achieve similar or even stronger photothermal effects, semiconductors can form multifunctional cellular interfaces to trigger photoluminescence ${ }^{98}$, for drug delivery ${ }^{98,101,160,161}$ or for photothermal cellular stimulation, and they can be designed to be biodegradable ${ }^{29,30}$. The electric capacitance of a cell membrane $\left(C_{m}\right)$ under heating (typical temperature change in an experiment is less than $4{ }^{\circ} \mathrm{C}$ ) can be modelled as $C_{m}(t)=C_{0}+C_{O \gamma} E(F(t) / \Delta t)$, where $\gamma$ is related to the temperaturedependent electrical capacitance change in membranes, $C_{0}$ is the initial capacitance, $E$ is the radiative pulse of energy and $F(t)$ describes the change in particle temperature as a function of time $t$. If experimental conditions allow large $\mathrm{d} C_{\mathrm{m}} / \mathrm{d} t$, sufficient capacitive currents can be injected into single cells to depolarize them. For example, mesostructured Si particles can be used for the photothermal stimulation of neurons by causing the firing of action potentials with a deterministic (that is, one light pulse causes the firing of one action potential) neuronal response of up to $15 \mathrm{~Hz}\left(\mathrm{REF}^{29}\right)$. A similar approach has been used for other 
organic-semiconductor-based modulation biointerfaces, for example, quinacridone-based ${ }^{162}$ and poly(3-hexylthiophene)-based ${ }^{163}$ interfaces.

In addition to extracellular modulation, the photothermal effect of semiconductor nanostructures can also be used for intracellular studies (FIG. 5a). For example, nanocrystalline Si nanowires that are internalized by glial cells can trigger a fast increase in calcium concentration upon a laser pulse ${ }^{30}$. This rapid heating can either generate ROS or transiently perforate calcium storage organelles, leading to an increase in cytosolic calcium concentration. Owing to intercellular interactions, photothermal modulation of the calcium concentration in glial cells can also elicit electrical activities in adjacent neurons and thus can also be applied for cytoskeletal transport studies in vitro ${ }^{30}$.

\section{Photoluminescent in vivo imaging}

Semiconductor-based photoluminescence can be exploited for the imaging of cells and tissues in vivo ${ }^{37,77,164}$ (FIG. 5a). A light signal is converted by a photoluminescent semiconductor into an output signal with a different wavelength (TABLE 2). Silicon is usually not photoluminescent because it is an indirect bandgap semiconductor. However, NIR photoluminescence in etched porous $\mathrm{Si}$ can be activated in an aqueous solution, which makes it particularly useful for in vivo imaging owing to the low levels of autofluorescence of biological tissue in the NIR range ${ }^{165}$. Its tunable photoluminescence, in combination with its structural, chemical and mechanical properties, has enabled the broad use of porous Si for imaging, drug delivery and diagnostic applications ${ }^{116,120,160,166-169}$.

In addition to surface defects, bulk doping can be used to introduce novel photoluminescent properties. Two-photon excitation has been widely used in biointerfaces; however, a threephoton process (that is, three photons are simultaneously absorbed for excitation) can be experimentally realized in biocompatible $\mathrm{Mn}^{2+}$-doped $\mathrm{ZnS}$ nanocrystals ${ }^{170}$. The doping enhances the Stokes shift, allowing the use of this material for in vivo tumour-targeted imaging ${ }^{170}$.

The photoluminescence of semiconducting carbon nanotubes can also be exploited for bioimaging in vivo, mostly because of their unique fluorescence emission within the second NIR window (NIR-II, wavelength $=1,100 \mathrm{~nm}-1,700 \mathrm{~nm})^{79}$. For example, carbon nanotubes have been used for through-skull fluorescence imaging, achieving a tissue depth of $>2 \mathrm{~mm}$ in a mouse brain with sub- $10 \mu \mathrm{m}$ resolution, owing to reduced photon scattering by the tissue in the NIR-II region ${ }^{80}$.

\section{Cytotoxicity}

Studies investigating the cytotoxicity of semiconductors are limited thus $\mathrm{far}^{4,171}$ and have mainly focused on $\mathrm{Si}\left(\operatorname{REFS}^{15,16,171}\right)$. Human blood plasma and serum contain silicon in the form of $\mathrm{Si}(\mathrm{OH})_{4}$ at concentrations of $<1 \mathrm{mg} \mathrm{Si} \mathrm{L}^{-1}\left(\mathrm{REF}^{172}\right)$, which is equivalent to an average dietary intake of $20-50 \mathrm{mg}$ per day ${ }^{173}$. Because of the natural presence of $\mathrm{Si}$ in the human body, only an injection of extensive amounts of soluble $\mathrm{Si}$ is expected to be toxic owing to, for example, renal tube blockage. Nevertheless, the biocompatibility of a particular Si material depends on the physicochemical properties of the surface of the material, the 
mode of administration, the biodistribution, the pharmacokinetics and the routes of excretion ${ }^{167}$. Therefore, careful examination of administered Si materials has to be performed to assess cytotoxicity and to enable clinical translation.

Many group III-V and group II-VI semiconductors contain elements (for example, As or Cd) that are potentially toxic ${ }^{174-176}$. However, the biocompatibility of semiconductors has been reported even in cell types that are highly sensitive to chemical perturbation (for example, neurons $)^{176-178}$. Meta-analyses can be applied to avoid a biased conclusion caused by the heterogeneity of published data and the often limited sample size. For example, the cytotoxicity of cadmium-containing semiconductor quantum dots (QDs) was evaluated using random forest regression models on the basis of information from literature data mining ${ }^{179}$. In this case study, more than 300 publications were mined, generating 1,741 QD toxicity data samples (each with a total of 24 qualitative and quantitative attributes). The study revealed that the cytotoxicity response to QDs is primarily related to intrinsic QD properties, such as diameter, surface ligand and shell and surface modification. Other features, such as exposure time, exposure concentration, assay type, cell type and cell origin, also had measurable relevance ${ }^{179}$. However, the meta-analysis tool is not yet standardized or fully developed. Machine learning algorithms and other meta-analysis techniques will certainly help to better predict the toxicity of engineered semiconductors in the future.

In addition to chemical cytotoxicity $4,174,175$, the mechanical mismatch between materials or devices and biological tissues can induce a severe inflammatory response ${ }^{180-182}$. Moreover, cytotoxicity can be induced by exogenous factors, such as light and voltage bias, which is currently underexplored but should be considered in the design of biointerfaces.

\section{Outlook and conclusions}

Cells not only function on the basis of biochemical and genetic pathways but also respond to and communicate through biophysical cues ${ }^{25,30}$, such as bioelectric and biomechanical signals. A variety of tools, for example, super-resolution microscopy and atomic force microscopy, have been developed to investigate the biophysical signalling pathways of cells. However, inorganic semiconductor materials and devices provide high performance and multifunctional modalities, scalability for multiplexed biointerfaces and compliance with the physical properties of biological tissues (TABLE 1), and they are biocompatible and can be designed to be biodegradable $1,25,30,81,92$.

Progress in semiconductor research, for example, related to energy materials $40,69,70,97,107,122-124,126,183,184$, has also opened up opportunities for biointerfaces and bio-physical studies, for example, the design of new semiconductor properties such as flexo-photovoltaic ${ }^{70}$ responses and room-temperature ductility ${ }^{71}$. Additionally, precise characterization techniques, such as high-speed atomic force microscopy, transient photoreflectance spectroscopy ${ }^{185}$, focused ion beam-scanning electron microscopy (FIBSEM) imaging tools ${ }^{43}$ and lattice light-sheet microscopy with adaptive optics ${ }^{186}$, can now be coupled with semiconductor-based probing. Moreover, theoretical and computational techniques ${ }^{184-187}$ can help to resolve the complex dynamics at the semiconductor-water interface, for example, for the investigation of non-covalent forces as well as the atomic and 
molecular dynamics within the double layer ${ }^{187}$. Finally, the identification of new material components and biological targets will expand future semiconductor-based biointerface applications.

\section{New material components}

Combining molecular biology techniques with inorganic semiconductor materials synthesis enables the study of crystallization processes at inorganic-organic interfaces ${ }^{188-192}$. For example, the genetic modification of cells ${ }^{192-193}$ can be applied in conjunction with inorganic semiconductor materials to present active motifs for biomineralization or for the biomimetic synthesis of inorganic semiconductor building blocks on cell surfaces ${ }^{27-194}$ and in intracellular domains ${ }^{192}$. Such multifunctional semiconductors, in combination with advanced microscopy and spectroscopy techniques, have the potential to reveal unprecedented information regarding fundamental biosynthetic mechanisms (for example, the transmembrane electron transfer pathway and its spatiotemporal dynamics). Inorganic semiconductors that are formed in situ at a cell membrane or within an organelle are further in close contact with the cell and therefore enable signal transduction between the cell and the inorganic material ${ }^{27}$. Thus, semiconductors can serve as markers for specific readouts (for example, to map bioelectric heterogeneity) or as modulators of a biological output (for example, to promote certain biosynthetic pathways).

Semiconductors could also potentially be designed with modulated composition and morphology, metastable crystal structures, controlled defect generation and new physicochemical properties by applying electrochemical deposition ${ }^{97,195}$, synthesis mediated by ionic liquids ${ }^{196}$, chemical conversion through ion exchange ${ }^{197}$ or a redox reaction ${ }^{198-200}$. Ionic liquids are low-temperature molten salts with high ion conductivity and low vapour pressure that do not decompose below $\sim 300{ }^{\circ} \mathrm{C}$. Therefore, ionic liquids could provide an ideal medium for the electrodeposition of semiconductors for enhanced light-induced activities at biointerfaces.

The Helmholtz double layer, the lateral heterogeneity of the space charge region ${ }^{39}$ (in semiconductors covered with isolated metal nanoparticles ${ }^{183}$ ) and surface states can also be engineered to achieve chemical control of the electrostatics and charge transfer behaviours ${ }^{38}$ and thus of the biophysical dynamics at the semiconductor-cell interface. In particular, the space charge region (band bending region) of a semiconductor covered with metal particles or patches ${ }^{183}$ is not only vertically distributed beneath the metal-semiconductor interface, which is typically described by an infinite metal-semiconductor interface model, but also laterally distributed along the semiconductor surface distant from the metal ${ }^{39}$. This feature results in electrostatic heterogeneity on the semiconductor surface ${ }^{183}$, enabling localized and efficient control of protein conformations and activities ${ }^{201,202}$, for example, of ion channels or cellular receptors.

Biocompatible catalysts can be designed to enable efficient signal transduction at the semiconductor surface, for example, by applying a biomimetic approach. Transition metal sulfides in nitrogenases ${ }^{203,204}$ and hydrogenases ${ }^{205}$ are excellent hydrogen evolving reaction (HER) catalysts. To mimic these reactions, $\mathrm{MoS}_{2}$ nanoparticles ${ }^{206}$ or cubane-type molecular metal sulfide clusters ${ }^{207}$ were used as semiconductor-based catalysts in HERs. A similar 
approach can be applied for biointerface studies. Finally, different types of catalysts ${ }^{126,195}$, including semiconductors ${ }^{206,207}$ and metal-coated semiconductors (for example, Si/Ti/Pt $\left(\mathrm{REF}^{126}\right)$ ), can be coupled to improve signal transduction across multiple length scales.

\section{New biological targets}

The cytoskeleton controls the active flow of signalling molecules inside cells and is crucial for numerous biological processes, such as neural signalling and carcino-genesis ${ }^{208,209}$. Microtubules, which are structurally polar filaments that serve as tracks for kinesin and dynein motor proteins, are an important part of the cytoskeleton. They not only contribute to the mechanical integrity of a cell but have also been proposed to be the source of electrodynamic activity owing to their electrical polarity, which causes normal vibration modes that generate an oscillating electric field ${ }^{210}$. Freestanding nano-structured semiconductors can produce a sustained or pulsed electrical and electrochemical effect, and therefore, cytoskeleton-semiconductor interfaces ${ }^{30,127}$ can be leveraged to assess the bioelectric heterogeneity inside a cell ${ }^{210}$. Such interfaces have been applied to uncover correlations between seemingly independent processes, for example, calcium wave propagations and cargo transport ${ }^{30}$.

Inorganic semiconductors can also be applied to investigate microorganisms, for example, the biophysics of microbial communities ${ }^{211-213}$. Semiconductor-bacteria interfaces have been used to mimic natural photosynthesis ${ }^{27,28}$. The rational design of semiconductor materials and devices is also being investigated for the manipulation of bacterial metabolism and long-range communication, using similar biophysical modulation mechanisms as for mammalian systems ${ }^{29,30,151}$.

Finally, semiconductors offer the opportunity to assess bioelectric heterogeneity in development and organ morphogenesis ${ }^{214,215}$. Freestanding semiconductor nanostructures or membranes, as well as flexible semiconductor electronics and optoelectronics, provide useful tools for the measurement of bioelectric activity, enabling movable electrical or electrochemical modulations and multiplexed control ${ }^{29,30,151}$ at a subcellular level, for example, to investigate the reaction-diffusion kinetics and bioelectric microenvironment during embryogenesis or organ regeneration.

\section{Acknowledgements}

This work is supported by the US National Institutes of Health (NIH NS101488), US Air Force Office of Scientific Research (AFOSR FA9550-18-1-0503), US Army Research Office (W911NF-18-1-0042) and US Office of Naval Research (ONRYIP, N000141612530; PECASE, N000141612958).

\section{References}

1. Zhang AQ \& Lieber CM Nano-bioelectronics. Chem. Rev 116, 215-257 (2016). [PubMed: 26691648]

2. Ning CZ, Dou LT \& Yang PD Bandgap engineering in semiconductor alloy nanomaterials with widely tunable compositions. Nat. Rev. Mater 2, 17070 (2017).

3. Zhang YH et al. Printing, folding and assembly methods for forming 3D mesostructures in advanced materials. Nat. Rev. Mater 2, 17019 (2017). 
4. Reiss P, Carriere M, Lincheneau C, Vaure L. \& Tamang S. Synthesis of semiconductor nanocrystals, focusing on nontoxic and earth-abundant materials. Chem. Rev 116, 10731-10819 (2016). [PubMed: 27391095]

5. Yu XG, Marks TJ \& Facchetti A. Metal oxides for optoelectronic applications. Nat. Mater 15, 383396 (2016). [PubMed: 27005918]

6. Lutz J, Schlangenotto H, Scheuermann U. \& DeDoncker R. Semiconductor Power Devices: Physics, Characteristics, Reliability (Springer-Verlag Berlin Heidelberg, 2011).

7. Pierret RF Semiconductor Device Fundamentals (Addison Wesley, 1996).

8. Sze SM \& Ng KK Physics of Semiconductor Devices (Wiley-Interscience, 1996).

9. Rivnay J. et al. Structural control of mixed ionic and electronic transport in conducting polymers. Nat. Commun 7, 11287 (2016). [PubMed: 27090156]

10. Xu J. et al. Highly stretchable polymer semiconductor films through the nanoconfinement effect. Science 355, 59-64 (2017). [PubMed: 28059762]

11. Bardeen J. \& Brattain WH The transistor, a semiconductor triode. Phys. Rev 74, 230-231 (1948).

12. Khambata AJ Introduction to Integrated Semiconductor Circuits (John Wiley and Sons, Inc., 1963).

13. Bergveld P. Development of an ion-sensitive solid-state device for neurophysiological measurements. IEEE Trans. Biomed. Eng 17, 70-71 (1970). [PubMed: 5441220]

14. Hafeman DG, Parce JW \& McConnell HM Light-addressable potentiometric sensor for biochemical systems. Science 240, 1182-1185 (1988). [PubMed: 3375810]

15. Canham LT Bioactive silicon structure fabrication through nanoetching techniques. Adv. Mater 7 , 1033-1037 (1995).

16. Kang SK et al. Bioresorbable silicon electronic sensors for the brain. Nature 530, 71-76 (2016). [PubMed: 26779949]

17. Bruchez M, Moronne M, Gin P, Weiss S. \& Alivisatos AP Semiconductor nanocrystals as fluorescent biological labels. Science 281, 2013-2016 (1998). [PubMed: 9748157]

18. Chan WCW \& Nie SM Quantum dot bioconjugates for ultrasensitive nonisotopic detection. Science 281, 2016-2018 (1998). [PubMed: 9748158]

19. Kim DH et al. Stretchable and foldable silicon integrated circuits. Science 320, 507-511 (2008).

This pioneering work provides a general strategy for the design and fabrication of high-performance $\mathrm{Si}$ circuits with reversible stretchability and deformability, applicable to brittle and fragile materials.

[PubMed: 18369106]

20. Fromherz P, Offenhausser A, Vetter T. \& Weis J. A neuron-silicon junction: a Retzius cell of the leech on an insulated-gate field-effect transistor. Science 252, 1290-1293 (1991). [PubMed: 1925540]

21. Patolsky F. et al. Detection, stimulation, and inhibition of neuronal signals with high-density nanowire transistor arrays. Science 313, 1100-1104 (2006). [PubMed: 16931757]

22. Tian BZ et al. Macroporous nanowire nanoelectronic scaffolds for synthetic tissues. Nat. Mater 11, 986-994 (2012).

This study is the first attempt to bridge bioelectronics with tissue engineering, using 3D, macroporous Si nanoelectronic scaffolds integrated with living cells for the real-time monitoring of local electrical and chemical environments.

[PubMed: 22922448]

23. Chow AY Electrical-stimulation of the rabbit retina with subretinal electrodes and high-density microphotodiode array implants. Invest. Ophthalmol. Vis. Sci 34, 835-835 (1993).

24. Kim TI et al. Injectable, cellular-scale optoelectronics with applications for wireless optogenetics. Science 340, 211-216 (2013).

This study is the first report of miniaturized optoelectronic devices for brain interfaces, using injected, wirelessly controlled inorganic microLED arrays to deliver optical stimuli for the control of animal behaviour. 
[PubMed: 23580530]

25. Tian BZ \& Lieber CM Synthetic nanoelectronic probes for biological cells and tissues. Annu. Rev. Anal. Chem 6, 31-51 (2013).

26. Parameswaran R. \& Tian BZ Rational design of semiconductor nanostructures for functional subcellular interfaces. Acc. Chem. Res 51, 1014-1022 (2018). [PubMed: 29668260]

27. Sakimoto KK, Wong AB \& Yang PD Self-photosensitization of nonphotosynthetic bacteria for solar-to-chemical production. Science 351, 74-77 (2016). [PubMed: 26721997]

28. Liu C. et al. Nanowire-bacteria hybrids for unassisted solar carbon dioxide fixation to value-added chemicals. Nano Lett 15, 3634-3639 (2015). [PubMed: 25848808]

29. Jiang YW et al. Heterogeneous silicon mesostructures for lipid-supported bioelectric interfaces. Nat. Mater 15, 1023-1030 (2016). [PubMed: 27348576]

30. Jiang YW et al. Rational design of silicon structures for optically controlled multiscale biointerfaces. Nat. Biomed. Eng 2, 508-521 (2018).

This study proposes a biology-guided rational design principle for Si structures for optically controlled biointerfaces to modulate biological activities, including the first demonstration of the non-genetic manipulation of animal motion with light.

[PubMed: 30906646]

31. Tian BZ et al. Three-dimensional, flexible nanoscale field-effect transistors as localized bioprobes. Science 329, 830-834 (2010).

This paper is the first to describe the design of Si-based 3D flexible electronics that can penetrate the cell and enable robust intracellular recordings of single cell action potentials.

[PubMed: 20705858]

32. Amit I. et al. Voices of biotech. Nat. Biotechnol 34, 270-275 (2016). [PubMed: 26963549]

33. Tian BZ et al. Roadmap on semiconductor-cell biointerfaces. Phys. Biol 15, 031002 (2018).

34. Lee J, Ozden I, Song YK \& Nurmikko AV Transparent intracortical microprobe array for simultaneous spatiotemporal optical stimulation and multichannel electrical recording. Nat. Methods 12, 1157-1162 (2015). [PubMed: 26457862]

35. Roder PB, Smith BE, Davis EJ \& Pauzauskie PJ Photothermal heating of nanowires. J. Phys. Chem. C 118, 1407-1416 (2014).

36. Chen H. \& Diebold G. Chemical generation of acoustic waves: a giant photoacoustic effect. Science 270, 963-966 (1995).

37. Jin D. et al. Nanoparticles for super-resolution microscopy and single-molecule tracking. Nat. Methods 15, 415-423 (2018). [PubMed: 29808018]

38. Bard AJ, Stratmann M. \& Licht S. Semiconductor Electrodes and Photoelectrochemistry Vol. 6 (Wiley-VCH, 2002).

39. Zhang Z. \& Yates JT Band bending in semiconductors: chemical and physical consequences at surfaces and interfaces. Chem. Rev 112, 5520-5551 (2012). [PubMed: 22783915]

40. Walter MG et al. Solar water splitting cells. Chem. Rev 110, 6446-6473 (2010).

This comprehensive review illustrates the fundamental principles underlying semiconductor-based photoelectrochemical devices and possible electron transfer mechanisms at semiconductor/ electrolyte junctions.

[PubMed: 21062097]

41. Zhao M. et al. Electrical signals control wound healing through phosphatidylinositol-3-OH kinasegamma and PTEN. Nature 442, 457-460 (2006). [PubMed: 16871217]

42. Plonsey R. \& Barr RC Bioelectricity: A Quantitative Approach (Springer-Verlag US, 2007).

43. Santoro $F$ et al. Revealing the cell-material interface with nanometer resolution by focused ion beam/scanning electron microscopy. ACS Nano 11, 8320-8328 (2017). 
This paper describes a high-resolution imaging technique for probing the cell-material interface, revealing that the cleft width between the cell membrane and a substrate is strongly influenced by the surface topographical curvatures.

[PubMed: 28682058]

44. Shoorideh K. \& Chui CO On the origin of enhanced sensitivity in nanoscale FET-based biosensors. Proc. Natl Acad. Sci. USA 111, 5111-5116 (2014). [PubMed: 24706861]

45. Zhao W. et al. Nanoscale manipulation of membrane curvature for probing endocytosis in live cells. Nat. Nanotechnol 12, 750-756 (2017). [PubMed: 28581510]

46. Patolsky F. et al. Electrical detection of single viruses. Proc. Natl Acad. Sci. USA 101, 1401714022 (2004). [PubMed: 15365183]

47. Stern E. et al. Importance of the debye screening length on nanowire field effect transistor sensors. Nano Lett 7, 3405-3409 (2007). [PubMed: 17914853]

48. Nakatsuka N. et al. Aptamer-field-effect transistors overcome debye length limitations for smallmolecule sensing. Science 362, 319-324 (2018). [PubMed: 30190311]

49. Kulkarni GS \& Zhong Z. Detection beyond the debye screening length in a high-frequency nanoelectronic biosensor. Nano Lett 12, 719-723 (2012). [PubMed: 22214376]

50. Lin TW et al. Label-free detection of protein-protein interactions using a calmodulin-modified nanowire transistor. Proc. Natl Acad. Sci. USA 107, 1047-1052 (2010). [PubMed: 20080536]

51. Stern E. et al. Label-free biomarker detection from whole blood. Nat. Nanotechnol 5, 138-142 (2010). [PubMed: 20010825]

52. Desai SB et al. $\mathrm{MoS}_{2}$ transistors with 1-nanometer gate lengths. Science 354, 99-102 (2016). [PubMed: 27846499]

53. Qiu C. et al. Scaling carbon nanotube complementary transistors to 5-nm gate lengths. Science 355, 271-276 (2017). [PubMed: 28104886]

54. Sarkar D. et al. A subthermionic tunnel field-effect transistor with an atomically thin channel. Nature 526, 91-95 (2015). [PubMed: 26432247]

55. Aharonovich I, Englund D. \& Toth M. Solid-state single-photon emitters. Nat. Photon 10, 631-641 (2016).

56. Tian B. et al. Coaxial silicon nanowires as solar cells and nanoelectronic power sources. Nature 449, 885-889 (2007). [PubMed: 17943126]

57. Savtchenko LP, Poo MM \& Rusakov DA Electrodiffusion phenomena in neuroscience: a neglected companion. Nat. Rev. Neurosci 18, 598-612 (2017). [PubMed: 28924257]

58. Yao J, Yan H. \& Lieber CM A nanoscale combing technique for the large-scale assembly of highly aligned nanowires. Nat. Nanotechnol 8, 329-335 (2013). [PubMed: 23603986]

59. Dragas J. et al. In vitro multi-functional microelectrode array featuring 59760 electrodes, 2048 electrophysiology channels, stimulation, impedance measurement, and neurotransmitter detection channels. IEEE J. Solid-State Circuits 52, 1576-1590 (2017). [PubMed: 28579632]

60. Abbott J. et al. CMOS nanoelectrode array for all-electrical intracellular electrophysiological imaging. Nat. Nanotechnol 12, 460-466 (2017). [PubMed: 28192391]

61. Abbott J, Ye TY, Ham D. \& Park H. Optimizing nanoelectrode arrays for scalable intracellular electrophysiology. Acc. Chem. Res 51, 600-608 (2018). [PubMed: 29437381]

62. Graham AHD, Robbins J, Bowen CR \& Taylor J. Commercialisation of CMOS integrated circuit technology in multi-electrode arrays for neuroscience and cell-based biosensors. Sensors 11, 4943-4971 (2011). [PubMed: 22163884]

63. Viventi J. et al. A conformal, bio-interfaced class of silicon electronics for mapping cardiac electrophysiology. Sci. Transl Med 2, 24ra22 (2010).

64. Tsai D, Sawyer D, Bradd A, Yuste R. \& Shepard KL A very large-scale microelectrode array for cellular-resolution electrophysiology. Nat. Commun 8, 1802 (2017). [PubMed: 29176752]

65 . Jun JJ et al. Fully integrated silicon probes for high-density recording of neural activity. Nature 551, 232-236 (2017). [PubMed: 29120427] 
66. Loudin JD, Cogan SF, Mathieson K, Sher A. \& Palanker DV Photodiode circuits for retinal prostheses. IEEE Trans. Biomed. Circuits Syst 5, 468-480 (2011). [PubMed: 23852178]

67. Mathieson K. et al. Photovoltaic retinal prosthesis with high pixel density. Nat. Photon 6, 391397(2012).

This study reports a system design of Si-based photovoltaic devices for retinal prosthetics, demonstrating high-resolution electrical stimulation of retinal responses through wireless actuation of multiplexed photodiode arrays.

68. Strukov DB \& Likharev KK Defect-tolerant architectures for nanoelectronic crossbar memories. J. Nanosci. Nanotechnol 7, 151-167 (2007). [PubMed: 17455481]

69. Narvaez J, Vasquez-Sancho F. \& Catalan G. Enhanced flexoelectric-like response in oxide semiconductors. Nature 538, 219-221 (2016). [PubMed: 27669023]

70. Yang MM, Kim DJ \& Alexe M. Flexo-photovoltaic effect. Science 360, 904-907 (2018). [PubMed: 29674433]

71. Shi X. et al. Room-temperature ductile inorganic semiconductor. Nat. Mater 17, 421-426 (2018). [PubMed: 29632407]

72. Zhang H. et al. Approaching the ideal elastic strain limit in silicon nanowires. Sci. Adv 2, e1501382 (2016).

73. Milo R. \& Phillips R. Cell Biology by the Numbers (Garland Science, Taylor \& Francis Group, 2016).

In this book, key numbers relevant to common cellular structures and events are discussed, serving as an important guidance for the rational design of biointerfaces.

74. Phillips R. \& Quake SR The biological frontier of physics. Phys. Today 59, 38-43 (2006).

75. Hong G, Yang X, Zhou T. \& Lieber CM Mesh electronics: a new paradigm for tissue-like brain probes. Curr. Opin. NeuroBiol 50, 33-41 (2018). [PubMed: 29202327]

76. Zhou W. et al. Long term stability of nanowire nanoelectronics in physiological environments. Nano Lett 14, 1614-1619 (2014). [PubMed: 24479700]

77. Mehlenbacher RD, Kolbl R, Lay A. \& Dionne JA Nanomaterials for in vivo imaging of mechanical forces and electrical fields. Nat. Rev. Mater 3, 17080 (2018).

78. Duan XJ, Fu TM, Liu J. \& Lieber CM Nanoelectronics-biology frontier: from nanoscopic probes for action potential recording in live cells to three-dimensional cyborg tissues. Nano Today 8, 351373 (2013). [PubMed: 24073014]

79. Hong G. et al. Multifunctional in vivo vascular imaging using near-infrared II fluorescence. Nat. Med 18, 1841-1846 (2012). [PubMed: 23160236]

80. Hong G. et al. Through-skull fluorescence imaging of the brain in a new near-infrared window. Nat. Photon 8, 723-730 (2014).

81. Rogers JA, Lagally MG \& Nuzzo RG Synthesis, assembly and applications of semiconductor nanomembranes. Nature 477, 45-53 (2011). [PubMed: 21886156]

82. Choi C. et al. Human eye-inspired soft optoelectronic device using high-density MoS2-graphene curved image sensor array. Nat. Commun 8, 1664 (2017) [PubMed: 29162854]

83. Liu C. et al. Rapid water disinfection using vertically aligned MoS2 nanofilms and visible light. Nat. Nanotechnol 11, 1098-1104 (2016). [PubMed: 27525474]

84. Parker SG et al. A photoelectrochemical platform for the capture and release of rare single cells. Nat. Commun 9, 2288 (2018). [PubMed: 29895867]

85. Luo ZQ et al. Atomic gold-enabled three-dimensional lithography for silicon mesostructures. Science 348, 1451-1455 (2015). [PubMed: 26113718]

86. Robinson JT et al. Vertical nanowire electrode arrays as a scalable platform for intracellular interfacing to neuronal circuits. Nat. Nanotechnol 7, 180-184 (2012). [PubMed: 22231664]

87. Colicos MA, Collins BE, Sailor MJ \& Goda Y. Remodeling of synaptic actin induced by photoconductive stimulation. Cell 107, 605-616 (2001). [PubMed: 11733060]

88. Lorach H. et al. Photovoltaic restoration of sight with high visual acuity. Nat. Med 21, 476-482 (2015). [PubMed: 25915832] 
89. Mandel Y. et al. Cortical responses elicited by photovoltaic subretinal prostheses exhibit similarities to visually evoked potentials. Nat. Commun 4, 1980 (2013). [PubMed: 23778557]

90. Park SI et al. Soft, stretchable, fully implantable miniaturized optoelectronic systems for wireless optogenetics. Nat. Biotechnol 33, 1280-1286 (2015). [PubMed: 26551059]

91. Dai XC, Hong GS, Gao T. \& Lieber CM Mesh nanoelectronics: seamless integration of electronics with tissues. Acc. Chem. Res 51, 309-318 (2018) [PubMed: 29381054]

92. Yan Z. et al. Three-dimensional mesostructures as high-temperature growth templates, electronic cellular scaffolds, and self-propelled microrobots. Proc. Natl Acad. Sci. USA 114, E9455-E9464 (2017). [PubMed: 29078394]

93. Kim DH et al. Epidermal electronics. Science 333, 838-843 (2011). [PubMed: 21836009]

94. Liu J. et al. Syringe-injectable electronics. Nat. Nanotechnol 10, 629-636 (2015). [PubMed: 26053995]

95. No YS et al. Encoding active device elements at nanowire tips. Nano Lett 16, 4713-4719 (2016). [PubMed: 27337041]

96. Mankin MN et al. Facet-selective epitaxy of compound semiconductors on faceted silicon nanowires. Nano Lett 15, 4776-4782 (2015). [PubMed: 26057208]

97. Kang D. et al. Electrochemical synthesis of photoelectrodes and catalysts for use in solar water splitting. Chem. Rev 115, 12839-12887 (2015). [PubMed: 26538328]

98. Park JH et al. Biodegradable luminescent porous silicon nanoparticles for in vivo applications. Nat. Mater 8, 331-336 (2009).

This study is the first report combining the tunable biodegradable and photoluminescent properties of porous Si for targeted drug delivery and imaging without causing toxic effects in vivo.

[PubMed: 19234444]

99. Orosco MM, Pacholski C. \& Sailor MJ Real-time monitoring of enzyme activity in a mesoporous silicon double layer. Nat. Nanotechnol 4, 255-258 (2009). [PubMed: 19350037]

100. Hochbaum AI, Gargas D, Hwang YJ \& Yang P. Single crystalline mesoporous silicon nanowires. Nano Lett 9, 3550-3554 (2009). [PubMed: 19824705]

101. Chiappini C. et al. Biodegradable silicon nanoneedles delivering nucleic acids intracellularly induce localized in vivo neovascularization. Nat. Mater 14, 532-539 (2015). [PubMed: 25822693]

102. Mirsky Y. et al. Optical biosensing of bacteria and cells using porous silicon based, photonic lamellar gratings. Appl. Phys. Lett 103, 033702 (2013).

103. Salonen J. \& Makila E. Thermally carbonized porous silicon and its recent applications. Adv. Mater 30, e1703819 (2018).

104. Chiappini C. et al. Biodegradable nanoneedles for localized delivery of nanoparticles in vivo: exploring the biointerface. ACS Nano 9, 5500-5509 (2015). [PubMed: 25858596]

105. Gu L. et al. In vivo time-gated fluorescence imaging with biodegradable luminescent porous silicon nanoparticles. Nat. Commun 4, 2326 (2013). [PubMed: 23933660]

106. Joo J. et al. Gated luminescence imaging of silicon nanoparticles. ACS Nano 9, 6233-6241 (2015). [PubMed: 26034817]

107. Sivula K. \& van de Krol R. Semiconducting materials for photoelectrochemical energy conversion. Nat. Rev. Mater 1, 15010 (2016).

108. Mak KF, Lee C, Hone J, Shan J. \& Heinz TF Atomically thin $\mathrm{MoS}_{2}$ : a new direct-gap semiconductor. Phys. Rev. Lett 105, 136805 (2010).

109. Erathodiyil N. \& Ying JY Functionalization of inorganic nanoparticles for bioimaging applications. Acc. Chem. Res 44, 925-935 (2011). [PubMed: 21648430]

110. Medintz IL, Uyeda HT, Goldman ER \& Mattoussi H. Quantum dot bioconjugates for imaging, labelling and sensing. Nat. Mater 4, 435-446 (2005). [PubMed: 15928695]

111. Lee JH, Zhang AQ, You SS \& Lieber CM Spontaneous internalization of cell penetrating peptidemodified nanowires into primary neurons. Nano Lett 16, 1509-1513 (2016). [PubMed: 26745653] 
112. Nel AE et al. Understanding biophysicochemical interactions at the nano-bio interface. Nat. Mater 8, 543-557 (2009). [PubMed: 19525947]

113. Lynch I. \& Dawson KA Protein-nanoparticle interactions. Nano Today 3, 40-47 (2008).

114. Mahmoudi M. et al. Protein-nanoparticle interactions: opportunities and challenges. Chem. Rev 111, 5610-5637 (2011). [PubMed: 21688848]

115. Hwang SW et al. A physically transient form of silicon electronics. Science 337, 1640-1644 (2012). [PubMed: 23019646]

116. Tasciotti E. et al. Mesoporous silicon particles as a multistage delivery system for imaging and therapeutic applications. Nat. Nanotechnol 3, 151-157 (2008). [PubMed: 18654487]

117. Hwang SW et al. Materials and fabrication processes for transient and bioresorbable highperformance electronics. Adv. Func. Mater 23, 4087-4093 (2013).

118. Li R. et al. An analytical model of reactive diffusion for transient electronics. Adv. Func. Mater 23, 3106-3114 (2013).

119. Hwang SW et al. Dissolution chemistry and biocompatibility of single-crystalline silicon nanomembranes and associated materials for transient electronics. ACS Nano 8, 5843-5851 (2014). [PubMed: 24684516]

120. Tzur-Balter A, Shatsberg Z, Beckerman M, Segal E. \& Artzi N. Mechanism of erosion of nanostructured porous silicon drug carriers in neoplastic tissues. Nat. Commun 6, 6208 (2015). [PubMed: 25670235]

121. Fang H. et al. Capacitively coupled arrays of multiplexed flexible silicon transistors for long-term cardiac electrophysiology. Nat. Biomed. Eng 1, 0038 (2017).

122. Kenney MJ et al. High-performance silicon photoanodes passivated with ultrathin nickel films for water oxidation. Science 342, 836-840 (2013). [PubMed: 24233719]

123. Hu S. et al. Amorphous $\mathrm{TiO}_{2}$ coatings stabilize $\mathrm{Si}, \mathrm{GaAs}$, and $\mathrm{GaP}$ photoanodes for efficient water oxidation. Science 344, 1005-1009 (2014). [PubMed: 24876492]

124. Chen YW et al. Atomic layer-deposited tunnel oxide stabilizes silicon photoanodes for water oxidation. Nat. Mater 10, 539-544 (2011).

This study describes a versatile surface treatment method enabling efficient and stable operation of Sibased photoelectrochemical devices through the decoupling of surface reactions from the underlying photovoltaic substrates.

[PubMed: 21685904]

125. Ji L. et al. Localized dielectric breakdown and antireflection coating in metal-oxidesemiconductor photoelectrodes. Nat. Mater 16, 127-131 (2017). [PubMed: 27820811]

126. Ji L. et al. A silicon-based photocathode for water reduction with an epitaxial SrTiO3 protection layer and a nanostructured catalyst. Nat. Nanotechnol 10, 84-90 (2015). [PubMed: 25437745]

127. Zimmerman JF et al. Cellular uptake and dynamics of unlabeled freestanding silicon nanowires. Sci. Adv 2, e1601039 (2016).

128. Xie C, Lin Z, Hanson L, Cui Y. \& Cui B. Intracellular recording of action potentials by nanopillar electroporation. Nat. Nanotechnol 7, 185-190 (2012). [PubMed: 22327876]

129. Dipalo M. et al. Plasmonic meta-electrodes allow intracellular recordings at network level on high-density CMOS-multi-electrode arrays. Nat. Nanotechnol 13, 965-971 (2018). [PubMed: 30104618]

130. Gomez-Martinez R. et al. Silicon chips detect intracellular pressure changes in living cells. Nat. Nanotechnol 8, 517-521 (2013). [PubMed: 23812188]

131. Zahid MU, Ma L, Lim SJ \& Smith AM Single quantum dot tracking reveals the impact of nanoparticle surface on intracellular state. Nat. Commun 9, 1830 (2018). [PubMed: 29739927]

132. Derfus AM, Chan WCW \& Bhatia SN Intracellular delivery of quantum dots for live cell labeling and organelle tracking. Adv. Mater 16, 961-966 (2004).

133. Qing Q. et al. Free-standing kinked nanowire transistor probes for targeted intracellular recording in three dimensions. Nat. Nanotechnol 9, 142-147 (2014). [PubMed: 24336402] 
134. Zheng GF, Patolsky F, Cui Y, Wang WU \& Lieber CM Multiplexed electrical detection of cancer markers with nanowire sensor arrays. Nat. Biotechnol 23, 1294-1301 (2005). [PubMed: 16170313]

135. Stern E. et al. Label-free immunodetection with CMOS-compatible semiconducting nanowires. Nature 445, 519-522 (2007). [PubMed: 17268465]

136. Lambacher A. et al. Identifying firing mammalian neurons in networks with high-resolution multi-transistor array (MTA). Appl. Phys. A 102, 1-11 (2011).

137. Hu Y, Xiang J, Liang G, Yan H. \& Lieber CM Sub-100 nanometer channel length Ge/Si nanowire transistors with potential for $2 \mathrm{THz}$ switching speed. Nano Lett 8, 925-930 (2008). [PubMed: 18251518]

138. Kotov NA et al. Nanomaterials for neural interfaces. Adv. Mater 21, 3970-4004 (2009).

139. Duan XJ et al. Intracellular recordings of action potentials by an extracellular nanoscale fieldeffect transistor. Nat. Nanotechnol 7, 174-179 (2012).

140. Gao RX et al. Outside looking in: nanotube transistor intracellular sensors. Nano Lett 12, 33293333 (2012). [PubMed: 22583370]

141. Jiang Z, Qing Q, Xie P, Gao RX \& Lieber CM Kinked p-n junction nanowire probes for high spatial resolution sensing and intracellular recording. Nano Lett 12, 1711-1716 (2012). [PubMed: 22309132]

142. Wrobel G. et al. Transmission electron microscopy study of the cell-sensor interface. J. R. Soc. Interface 5, 213-222 (2008). [PubMed: 17609177]

143. Braun D. \& Fromherz P. Imaging neuronal seal resistance on silicon chip using fluorescent voltage-sensitive dye. Biophys. J. 87, 1351-1359 (2004). [PubMed: 15298937]

144. Mimee M. et al. An ingestible bacterial-electronic system to monitor gastrointestinal health. Science 360, 915-918 (2018).

This study describes the first prototype of an ingestible optoelectronic device for remote sensing of the gastrointestinal microenvironment by detecting and transmitting engineered bacterial bioluminescence signals to report local bleeding and biomolecular dynamics.

[PubMed: 29798884]

145. Iddan G, Meron G, Glukhovsky A. \& Swain P. Wireless capsule endoscopy. Nature 405, 417-418 (2000).

146. Lu LY et al. Wireless optoelectronic photometers for monitoring neuronal dynamics in the deep brain. Proc. Natl Acad. Sci. USA 115, E1374-E1383 (2018). [PubMed: 29378934]

147. Wang LL et al. Photovoltaic retinal prosthesis: implant fabrication and performance. J. Neural Eng 9, 046014 (2012).

148. Ghezzi D. et al. A hybrid bioorganic interface for neuronal photoactivation. Nat. Commun 2, 166 (2011). [PubMed: 21266966]

149. Savchenko A. et al. Graphene biointerfaces for optical stimulation of cells. Sci. Adv 4, eaat0351 (2018).

150. Rand D. et al. Direct electrical neurostimulation with organic pigment photocapacitors. Adv. Mater 30, e1707292 (2018).

151. Parameswaran R. et al. Photoelectrochemical modulation of neuronal activity with free-standing coaxial silicon nanowires. Nat. Nanotechnol 13, 260-266 (2018). [PubMed: 29459654]

152. Tang J. et al. Nanowire arrays restore vision in blind mice. Nat. Commun 9, 786 (2018). [PubMed: 29511183]

153. Brown KA et al. Light-driven dinitrogen reduction catalyzed by a CdS:nitrogenase MoFe protein biohybrid. Science 352, 448-450 (2016). [PubMed: 27102481]

154. Clarke SJ et al. Photophysics of dopamine-modified quantum dots and effects on biological systems. Nat. Mater 5, 409-417 (2006). [PubMed: 16617348]

155. Claassens NJ, Sousa DZ, dos Santos V, de Vos WM \& van der Oost J. Harnessing the power of microbial autotrophy. Nat. Rev. MicroBiol 14, 692-706 (2016). [PubMed: 27665719]

156. Courtney $\mathrm{CM}$ et al. Photoexcited quantum dots for killing multidrug-resistant bacteria. Nat. Mater 15, 529-534 (2016). [PubMed: 26779882] 
157. Bossio C. et al. Photocatalytic activity of polymer nanoparticles modulates intracellular calcium dynamics and reactive oxygen species in HEK-293 cells. Front. Bioeng. Biotechnol 6, 114 (2018). [PubMed: 30211158]

158. Siuda ER et al. Optodynamic simulation of beta-adrenergic receptor signalling. Nat. Commun 6 , 8480 (2015). [PubMed: 26412387]

159. Ding H. et al. Microscale optoelectronic infrared-to-visible upconversion devices and their use as injectable light sources. Proc. Natl Acad. Sci. USA 115, 6632-6637 (2018). [PubMed: 29891705]

160. Hussain S. et al. Antibiotic-loaded nanoparticles targeted to the site of infection enhance antibacterial efficacy. Nat. Biomed. Eng 2, 95-103 (2018). [PubMed: 29955439]

161. Kim B. et al. Immunogene therapy with fusogenic nanoparticles modulates macrophage response to staphylococcus aureus. Nat. Commun 9, 1969 (2018). [PubMed: 29773788]

162. Sytnyk M. et al. Cellular interfaces with hydrogen-bonded organic semiconductor hierarchical nanocrystals. Nat. Commun 8, 91 (2017). [PubMed: 28733618]

163. Martino N. et al. Photothermal cellular stimulation in functional bio-polymer interfaces. Sci. Rep 5, 8911 (2015). [PubMed: 25753132]

164. Efros AL et al. Evaluating the potential of using quantum dots for monitoring electrical signals in neurons. Nat. Nanotechnol 13, 278-288 (2018). [PubMed: 29636589]

165. Hong G, Antaris AL \& Dai H. Near-infrared fluorophores for biomedical imaging. Nat. Biomed. Eng 1, 0010 (2017).

166. Xu R. et al. An injectable nanoparticle generator enhances delivery of cancer therapeutics. Nat. Biotechnol 34, 414-418 (2016). [PubMed: 26974511]

167. Bimbo LM et al. Biocompatibility of thermally hydrocarbonized porous silicon nanoparticles and their biodistribution in rats. ACS Nano 4, 3023-3032 (2010). [PubMed: 20509673]

168. Vilensky R, Bercovici M. \& Segal E. Oxidized porous silicon nanostructures enabling electrokinetic transport for enhanced DNA detection. Adv. Func. Mater 25, 6725-6732 (2015).

169. Jane A, Dronov R, Hodges A. \& Voelcker NH Porous silicon biosensors on the advance. Trends Biotechnol 27, 230-239 (2009). [PubMed: 19251329]

170. Yu JH et al. High-resolution three-photon biomedical imaging using doped ZnS nanocrystals. Nat. Mater 12, 359-366 (2013). [PubMed: 23416726]

171. Montalti M, Cantelli A. \& Battistelli G. Nanodiamonds and silicon quantum dots: ultrastable and biocompatible luminescent nanoprobes for long-term bioimaging. Chem. Soc. Rev 44, 48534921 (2015). [PubMed: 26051500]

172. Bercowy GM, Vo H. \& Rieders F. Silicon analysis in biological specimens by direct-current plasma-atomic emission-spectroscopy. J. Anal. Toxicol 18, 46-48 (1994). [PubMed: 8127084]

173. Pennington JAT Silicon in foods and diets. Food Addit. Contam 8, 97-118 (1991). [PubMed: 2015936]

174. Srivastava V, Gusain D. \& Sharma YC Critical review on the toxicity of some widely used engineered nanoparticles. Ind. Eng. Chem. Res 54, 6209-6233 (2015).

175. Wu YL et al. Biophysical responses upon the interaction of nanomaterials with cellular interfaces. Acc. Chem. Res 46, 782-791 (2013). [PubMed: 23194178]

176. Gautam V. et al. Engineering highly interconnected neuronal networks on nanowire scaffolds. Nano Lett 17, 3369-3375 (2017). [PubMed: 28437614]

177. Hallstrom W. et al. Gallium phosphide nanowires as a substrate for cultured neurons. Nano Lett 7 , 2960-2965 (2007). [PubMed: 17880143]

178. Piret G, Perez MT \& Prinz CN Neurite outgrowth and synaptophysin expression of postnatal CNS neurons on GaP nanowire arrays in long-term retinal cell culture. Biomaterials 34, 875-887 (2013). [PubMed: 23131535]

179. Oh E. et al. Meta-analysis of cellular toxicity for cadmium-containing quantum dots. Nat. Nanotechnol 11, 479-486 (2016). [PubMed: 26925827]

180. Lacour SP, Courtine G. \& Guck J. Materials and technologies for soft implantable neuroprostheses. Nat. Rev. Mater 1, 16063 (2016). 
181. Salatino JW, Ludwig KA, Kozai TDY \& Purcell EK Glial responses to implanted electrodes in the brain. Nat. Biomed. Eng 1 , 862-877 (2017). [PubMed: 30505625]

182. Chen R, Canales A. \& Anikeeva P. Neural recording and modulation technologies. Nat. Rev. Mater 2, 16093 (2017). [PubMed: 31448131]

183. Hill JC, Landers AT \& Switzer JA An electrodeposited inhomogeneous metal-insulatorsemiconductor junction for efficient photoelectrochemical water oxidation. Nat. Mater 14, 1150 1155 (2015). [PubMed: 26366847]

184. Pham TA, Ping Y. \& Galli G. Modelling heterogeneous interfaces for solar water splitting. Nat. Mater 16, 401-408 (2017).

In this Review, the structural and electronic properties at photoelectrochemical device interfaces and first-principles-based approaches for predicting interfacial properties are discussed.

[PubMed: 28068314]

185. Yang Y. et al. Semiconductor interfacial carrier dynamics via photoinduced electric fields. Science 350, 1061-1065 (2015). [PubMed: 26612947]

186. Liu TL et al. Observing the cell in its native state: imaging subcellular dynamics in multicellular organisms. Science 360, 284-284 (2018).

187. Stamenkovic VR, Strmcnik D, Lopes PP \& Markovic NM Energy and fuels from electrochemical interfaces. Nat. Mater 16, 57-69 (2017).

188. Mann S. et al. Crystallization at inorganic-organic interfaces: biominerals and biomimetic synthesis. Science 261, 1286-1292 (1993). [PubMed: 17731856]

189. Gower LB Biomimetic model systems for investigating the amorphous precursor pathway and its role in biomineralization. Chem. Rev 108, 4551-4627 (2008). [PubMed: 19006398]

190. Shtukenberg AG, Ward MD \& Kahr B. Crystal growth with macromolecular additives. Chem. Rev 117, 14042-14090 (2017). [PubMed: 29165999]

191. Hildebrand M. Diatoms, biomineralization processes, and genomics. Chem. Rev 108, 4855-4874 (2008). [PubMed: 18937513]

192. Kolinko I. et al. Biosynthesis of magnetic nanostructures in a foreign organism by transfer of bacterial magnetosome gene clusters. Nat. Nanotechnol 9, 193-197 (2014). [PubMed: 24561353]

193. Chen AY et al. Synthesis and patterning of tunable multiscale materials with engineered cells. Nat. Mater 13, 515-523 (2014). [PubMed: 24658114]

194. Niu J. et al. Engineering live cell surfaces with functional polymers via cytocompatible controlled radical polymerization. Nat. Chem 9, 537-545 (2017). [PubMed: 28537595]

195. Ozel T. et al. Electrochemical deposition of conformal and functional layers on high aspect ratio silicon micro/nanowires. Nano Lett 17, 4502-4507 (2017). [PubMed: 28621537]

196. Armand M, Endres F, MacFarlane DR, Ohno H. \& Scrosati B. Ionic-liquid materials for the electrochemical challenges of the future. Nat. Mater 8, 621-629 (2009). [PubMed: 19629083]

197. Holtus T. et al. Shape-preserving transformation of carbonate minerals into lead halide perovskite semiconductors based on ion exchange/insertion reactions. Nat. Chem 10, 740-745 (2018). [PubMed: 29867120]

198. Bao ZH et al. Chemical reduction of three-dimensional silica micro-assemblies into microporous silicon replicas. Nature 446, 172-175 (2007). [PubMed: 17344850]

199. Moreno C. et al. Bottom-up synthesis of multifunctional nanoporous graphene. Science 360, 199203 (2018). [PubMed: 29650671]

200. Kim DY, Stefanoski S, Kurakevych OO \& Strobel TA Synthesis of an open-framework allotrope of silicon. Nat. Mater 14, 169-173 (2015). [PubMed: 25401923]

201. Bezanilla F. How membrane proteins sense voltage. Nat. Rev. Mol. Cell. Biol 9, 323-332 (2008).

In this Review, the basic principles of how membrane proteins sense transmembrane voltage and how such responses transmit into cellular function are discussed.

[PubMed: 18354422]

202. Swartz KJ Sensing voltage across lipid membranes. Nature 456, 891-897 (2008). [PubMed: 19092925] 
203. Hoffman BM, Lukoyanov D, Dean DR \& Seefeldt LC Nitrogenase: a draft mechanism. Acc. Chem. Res 46, 587-595 (2013). [PubMed: 23289741]

204. Lee SC, Lo W. \& Holm RH Developments in the biomimetic chemistry of cubane-type and higher nuclearity iron-sulfur clusters. Chem. Rev 114, 3579-3600 (2014). [PubMed: 24410527]

205. Simmons TR, Berggren G, Bacchi M, Fontecave M. \& Artero V. Mimicking hydrogenases: from biomimetics to artificial enzymes. Coordin. Chem. Rev 270, 127-150 (2014).

206. Jaramillo TF et al. Identification of active edge sites for electrochemical $\mathrm{H}_{2}$ evolution from $\mathrm{MoS}_{2}$ nanocatalysts. Science 317, 100-102 (2007). [PubMed: 17615351]

207. Jaramillo TF et al. Hydrogen evolution on supported incomplete cubane-type $\left[\mathrm{Mo}_{3} \mathrm{~S}_{4}\right]^{4+}$ electrocatalysts. J. Phys. Chem. C 112, 17492-17498 (2008).

208. Goldenring JR A central role for vesicle trafficking in epithelial neoplasia: intracellular highways to carcinogenesis. Nat. Rev. Cancer 13, 813-820 (2013). [PubMed: 24108097]

209. Nirschl JJ, Ghiretti AE \& Holzbaur ELF The impact of cytoskeletal organization on the local regulation of neuronal transport. Nat. Rev. Neurosci 18, 585-597 (2017). [PubMed: 28855741]

210. Havelka D, Cifra M, Kucera O, Pokorny J. \& Vrba J. High-frequency electric field and radiation characteristics of cellular microtubule network. J. Theor. Biol 286, 31-40 (2011). [PubMed: 21782830]

211. Humphries J. et al. Species-independent attraction to biofilms through electrical signaling. Cell 168, 200-209 (2017). [PubMed: 28086091]

212. Liu J. et al. Metabolic co-dependence gives rise to collective oscillations within biofilms. Nature 523, 550-554 (2015). [PubMed: 26200335]

213. Prindle A. et al. Ion channels enable electrical communication in bacterial communities. Nature 527, 59-63 (2015).

This article is the first to report $\mathrm{K}+$-ion-channel-mediated long-range electrical signalling within bacterial biofilms, marking the beginning of bioelectric studies of microbial communities.

[PubMed: 26503040]

214. Pai VP et al. HCN2 rescues brain defects by enforcing endogenous voltage pre-patterns. Nat. Commun 9, 998 (2018). [PubMed: 29519998]

215. Lobikin M. et al. Serotonergic regulation of melanocyte conversion: a bio-electrically regulated network for stochastic all-or-none hyperpigmentation. Sci. Signal. 8, ra99 (2015).

216. Gratzel M. Photoelectrochemical cells. Nature 414, 338-344 (2001). [PubMed: 11713540]

217. Kudo A. \& Miseki Y. Heterogeneous photocatalyst materials for water splitting. Chem. Soc. Rev 38, 253-278 (2009). [PubMed: 19088977]

218. Walsh A. et al. Nature of the band gap of $\mathrm{In}_{2} \mathrm{O}_{3}$ revealed by first-principles calculations and $\mathrm{x}$-ray spectroscopy. Phys. Rev. Lett 100, 167402 (2008).

219. Kormanyos A. et al. Solution combustion synthesis, characterization, and photoelectrochemistry of $\mathrm{CuNb}_{2} \mathrm{O} 6$ and $\mathrm{ZnNb}_{2} \mathrm{O} 6$ nanoparticles. J. Phys. Chem. C 120, 1(5024-16034 (2016).

220. Fuechsle M. et al. Spectroscopy of few-electron single-crystal silicon quantum dots. Nat. Nanotechnol 5, 502-505 (2010). [PubMed: 20495552]

221. Wu Y. et al. Controlled growth and structures of molecular-scale silicon nanowires. Nano Lett 4 , 433-436 (2004).

222. Fang Y. et al. Alloy-assisted deposition of three-dimensional arrays of atomic gold catalyst for crystal growth studies. Nat. Commun 8, 2014 (2017). [PubMed: 29222439]

223. Guan L, Suenaga K. \& Iijima S. Smallest carbon nanotube assigned with atomic resolution accuracy. Nano Lett 8, 459-462 (2008). [PubMed: 18186659]

224. Kang K. et al. High-mobility three-atom-thick semiconducting films with wafer-scale homogeneity. Nature 520, 656-660 (2015). [PubMed: 25925478]

225. Sudhof TC The presynaptic active zone. Neuron 75, 11-25 (2012). [PubMed: 22794257]

226. Lee YK et al. Dissolution of monocrystalline silicon nanomembranes and their use as encapsulation layers and electrical interfaces in water-soluble electronics. ACS Nano 11, 1256212572 (2017). [PubMed: 29178798] 
227. Photonics Hamamatsu. Technical note: ORCA-Flash4.0 V3: digital CMOS camera. Hamamatsu https://www.hamamatsu.com/resources/pdf/sys/SCAS0134E_C13440-20CU_tec.pdf (2018).

228. Jenssen S, Gracely EJ \& Sperling MR How long do most seizures last? A systematic comparison of seizures recorded in the epilepsy monitoring unit. Epilepsia 47, 1499-1503 (2006). [PubMed: 16981866]

229. Connors BW \& Gutnick MJ Intrinsic firing patterns of diverse neocortical neurons. Trends Neurosci 13, 99-104 (1990). [PubMed: 1691879]

230. Levin M. \& Stevenson CG Regulation of cell behavior and tissue patterning by bioelectrical signals: challenges and opportunities for biomedical engineering. Annu. Rev. Biomed. Eng 14, 295-323 (2012). [PubMed: 22809139]

231. Reimann MW et al. A biophysically detailed model of neocortical local field potentials predicts the critical role of active membrane currents. Neuron 79, 375-390 (2013). [PubMed: 23889937]

232. Gittes F. Flexural rigidity of microtubules and actin filaments measured from thermal fluctuations in shape. J. Cell Biol 120, 923-934 (1993). [PubMed: 8432732]

233. Wen Q. \& Janmey PA Polymer physics of the cytoskeleton. Curr. Opin. Solid State Mater. Sci 15, 177-182 (2011). [PubMed: 22081758]

234. Allendorf MD, Melius CF, Ho P. \& Zachariah MR Theoretical study of the thermochemistry of molecules in the Si-O-H system. J. Phys. Chem 99, 15285-15293 (1995).

235. Feldbauer K. et al. Channelrhodopsin-2 is a leaky proton pump. Proc. Natl Acad. Sci. USA 106, 12317-12322 (2009). [PubMed: 19590013]

236. Laughlin SB, de Ruyter van Steveninck RR \& Anderson JC The metabolic cost of neural information. Nat. Neurosci 1, 36-41 (1998). [PubMed: 10195106] 


\section{Box 1 |}

\section{Photoelectric, photovoltaic, photocapacitive, photoelectrochemical and photoconductive effect Photoelectric effect}

Photoelectric effect

Photoelectric effect is a phenomenon occurring on the surface of dry-state materials in a vacuum, in which electrons are emitted from the material upon light illumination. This effect is not unique to semiconductors but also occurs in metals, which can emit photoelectrons if the incident photon energy exceeds the metal work function.

Photovoltaic effect

Photovoltaic effect is a unique property of a semiconductor, occurring as a bulk process. Upon light illumination, excess charge carriers are generated inside the semiconductor. Charge carrier movements cause potential differences inside the material, which can provide all the electrical power required for optoelectronic stimulation of cells or tissues. If the semiconductor is in contact with a liquid electrolyte, carriers at the semiconductor surface can trigger either capacitive or Faradaic processes, depending on the nature of the solid-liquid interface.

Photocapacitive effect

Photocapacitive effect originates from the additional charge carriers generated in semiconductors upon light illumination. At the solid-liquid interface, charge injection into the solution is either inhibited or extremely slow. Ions with a charge opposite to the light-produced excessive electrons or holes are attracted to the semiconductor surface, causing a transient ionic current flow that capacitively charges the Helmholtz double layer at the semiconductor-electrolyte interface.

Photoelectrochemical effect

Photoelectrochemical effect has an origin similar to that of the photocapacitive effect and is mainly based on Faradaic processes. In a photoelectrochemical device, charge injection into the solution is possible at the solid-liquid interface. Therefore, light-produced excessive electrons or holes can cause reduction or oxidation of a solution species, that is, cathodic or anodic reactions.

Photoconductive effect

Photoconductive effect is the light-induced increase in charge injection at the devicesaline interface, that is, a photoelectrochemical effect. A semiconductor device exploiting the photoconductive effect can have two different interconnect configurations. In one configuration, one electrical contact terminal is placed on the semiconductor and controlled by an external power source $66,67,87$, and the other terminal is placed in the bath solution. In the other configuration, the semiconductor can function as a phototransistor and is passivated in saline ${ }^{82}$. Light is used to modulate carrier concentration and semiconductor conductance. The change in conductance is then coupled to a stimulation electrode through additional circuits to provide an interface with cells or tissues in saline $^{82}$. In both configurations, the semiconductor-electrolyte ${ }^{87}$ or electrode- 
electrolyte ${ }^{66,67,82}$ junction conduction is altered by shining light on the semiconductor phase, and the semiconductors can either be uniform ${ }^{82,87}$ or contain diode junctions ${ }^{66,67}$. 


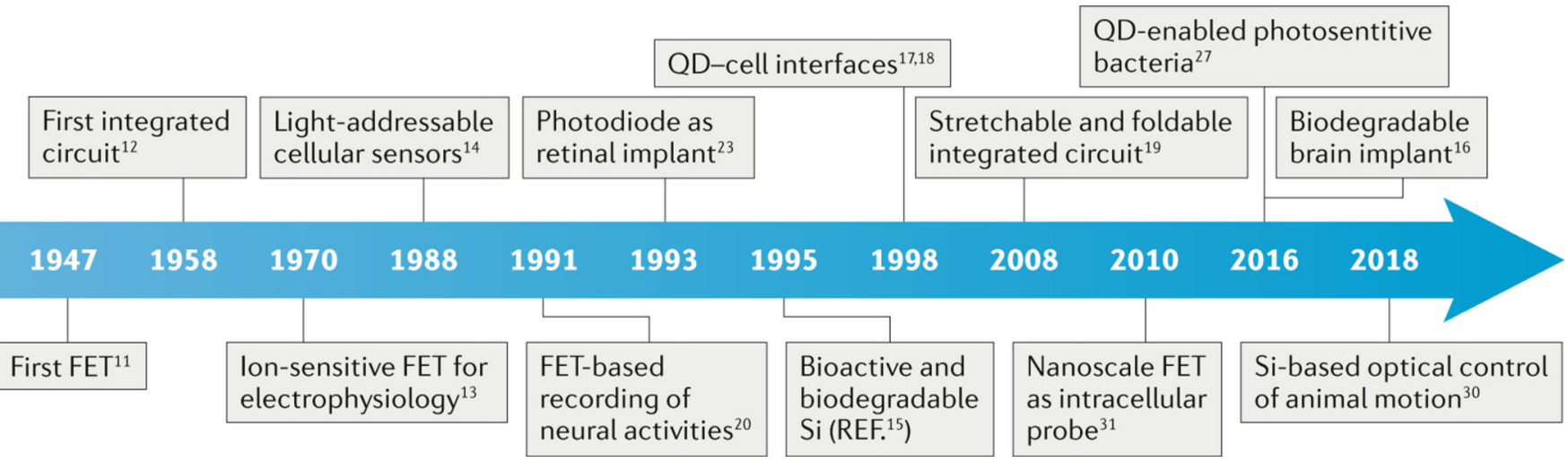

Fig. 1 . Milestones of inorganic semiconductor devices for biological studies.

FET, field-effect transistor; QD, quantum dot. 
a

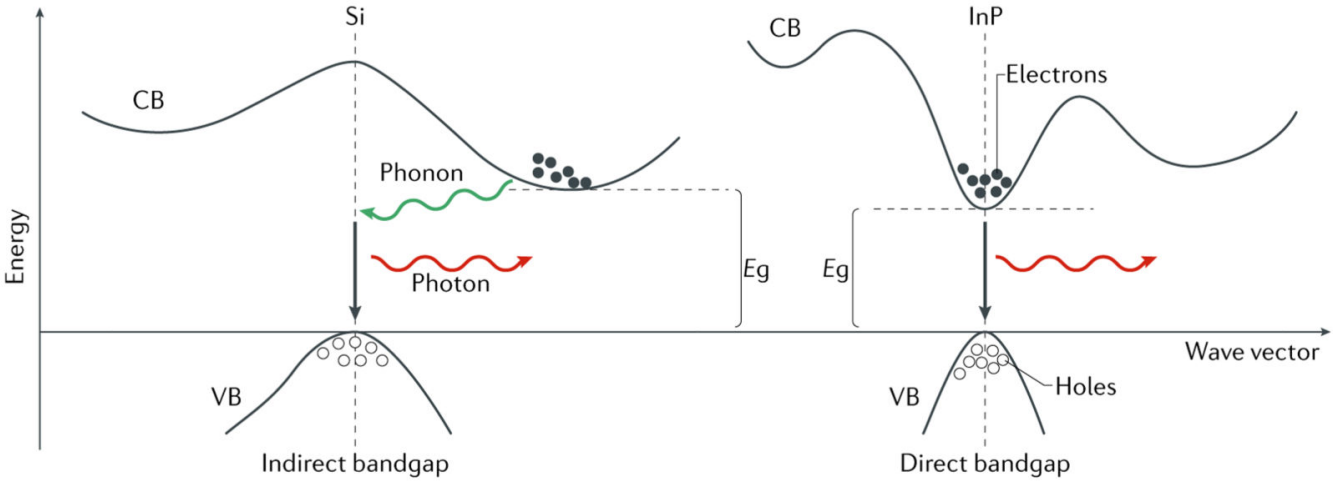

b

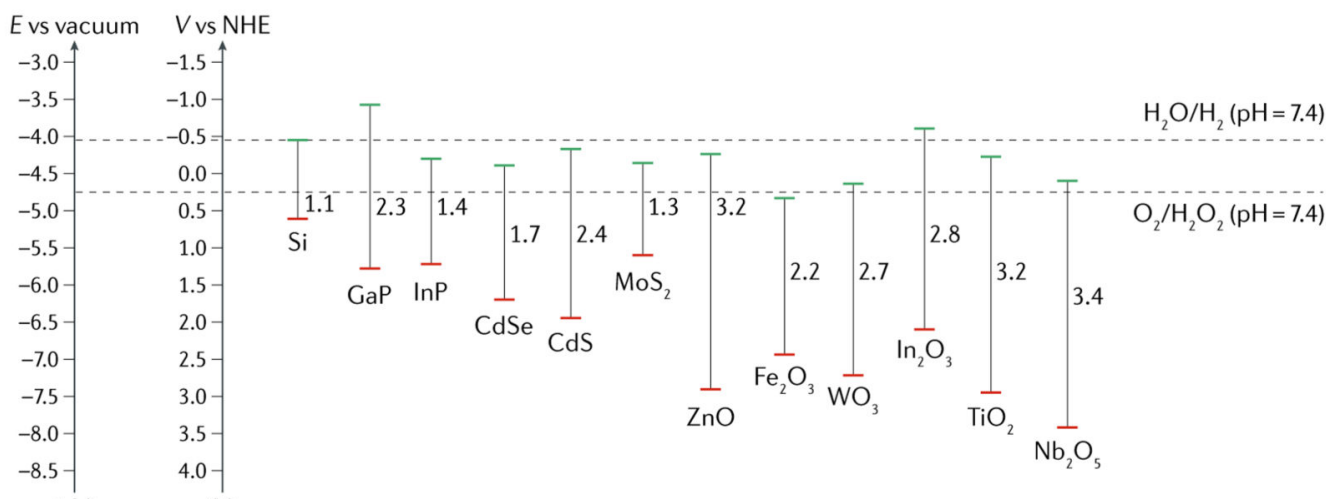

(V)
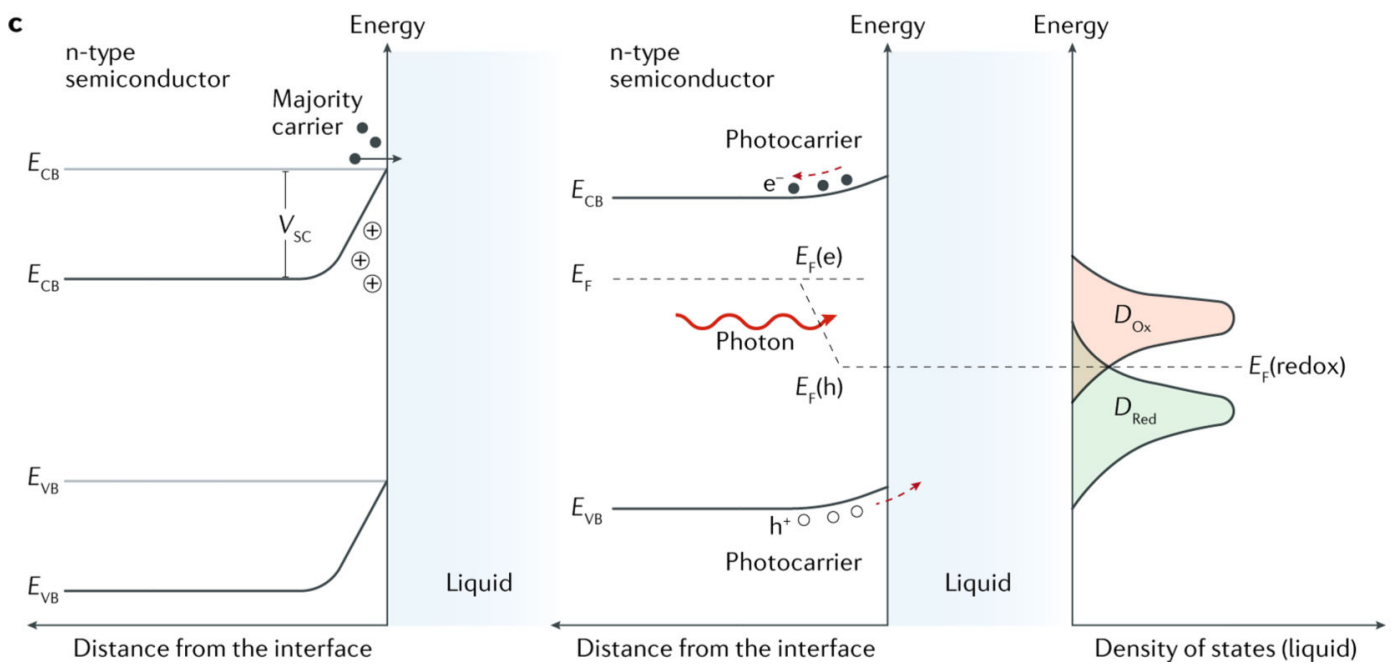

Fig. 2 |. Material physics at the semiconductor-saline interface.

a Band structures of inorganic semiconductors. The bandgap $E_{\mathrm{g}}$ is defined as the energy difference between the lowest point of the conduction band (CB) and the highest point of the valence band (VB). A semiconductor can have either an indirect or a direct bandgap. In an indirect bandgap semiconductor (for example, $\mathrm{Si}$ ), the $\mathrm{VB}$ and $\mathrm{CB}$ edges are not aligned in the momentum space and thus the electrons in the VB cannot be directly excited to the $\mathrm{CB}$ without the assistance of phonons. In a direct bandgap semiconductor (for example, InP), the electron transfer between the VB and the CB occurs directly through the absorption or 
emission of photons. The two band structures are not to scale. $\mathbf{b} \mid$ Band edge positions of different inorganic semiconductors with respect to vacuum level and normal hydrogen electrode (NHE) potential. The redox potentials of the $\mathrm{H}_{2} \mathrm{O} / \mathrm{H}_{2}$ and $\mathrm{O}_{2} / \mathrm{H}_{2} \mathrm{O}_{2}$ couples at physiological $\mathrm{pH}$ are drawn as references (dashed lines). Band edge positions and bandgap energies are based on data from the literature ${ }^{83,97,216-219}$. $\mathbf{c} \mid$ Band bending at semiconductor-saline interfaces. Band bending occurs if a semiconductor is immersed in a saline solution, as the charge flow between the solid and liquid phases aims to reach an equilibrium in terms of Fermi levels $\left(E_{\mathrm{F}}=E_{\mathrm{F}}(\right.$ redox $\left.)\right)$. $E_{\mathrm{CB}}$ and $E_{\mathrm{VB}}$ are the energies of the $\mathrm{CB}$ and $\mathrm{VB}$ edges, respectively. $V_{\mathrm{SC}}$ is the semiconductor barrier height at the depletion layer. Illumination of the semiconductor causes different energy distributions of the photogenerated carriers (that is, electrons and holes $\left(\mathrm{h}^{+}\right)$) occupying the corresponding quasi-Fermi levels $E_{\mathrm{F}}(\mathrm{e})$ and $E_{\mathrm{F}}(\mathrm{h})$. Swept by the built-in electric field, photocarriers can be injected into the solution for surface electrochemical reactions with the corresponding redox species. $D_{\mathrm{Ox}}$ and $D_{\mathrm{Red}}$ are the densities of states for oxidants and reductants in the saline solution, respectively. 
a FET

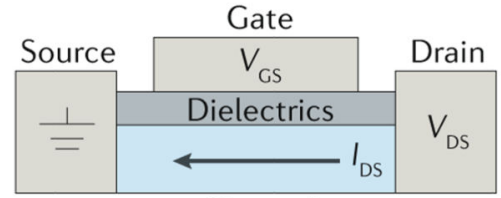

Channel

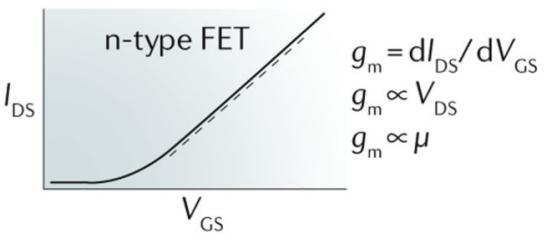

b $\mathrm{p}-\mathrm{n}$ diode

n-type p-type

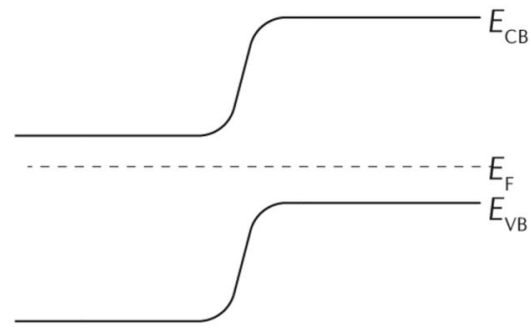

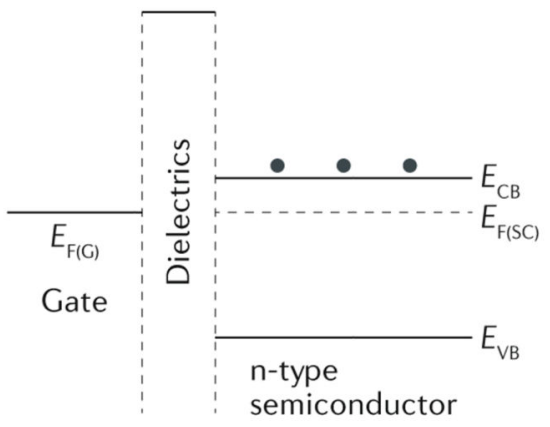

c Photovoltaic device

n-type

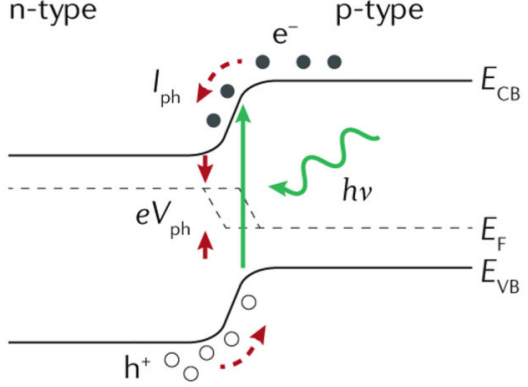

Minority carrier

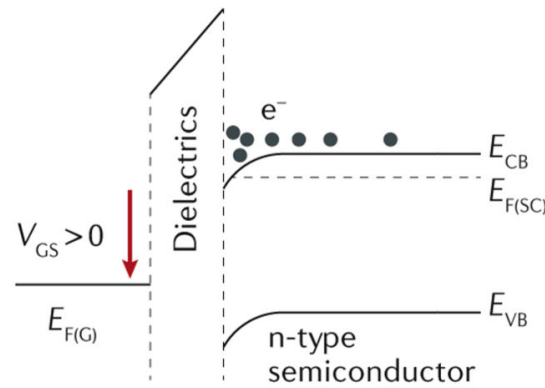

d LED

n-type p-type

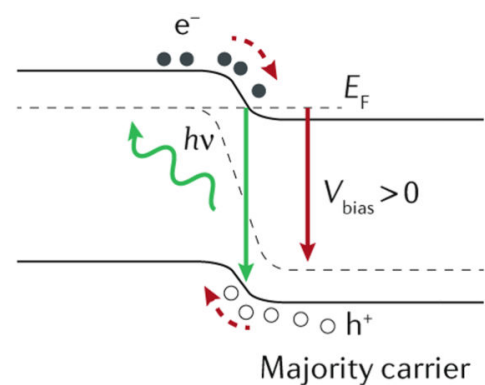

Fig. 3 |. Operation principles of inorganic semiconductor devices.

a $\mid$ A field-effect transistor (FET) device consists of a semiconductor channel, a dielectric layer and three electrodes (source, drain and gate). The gate-source voltage $\left(\mathrm{V}_{\mathrm{GS}}\right)$ can modulate the drain-source current $\left(I_{\mathrm{DS}}\right)$. The transconductance $g_{\mathrm{m}} \propto$ typically follows $g_{\mathrm{m}} \propto$ $\mu V_{\mathrm{DS}} \cdot \mu$ is the charge carrier mobility, and $\mathrm{V}_{\mathrm{DS}}$ is the drain-source voltage. $E_{\mathrm{F}(\mathrm{G})}$ and $\mathrm{E}_{\mathrm{F}(\mathrm{SC})}$ are the Fermi levels of the gate and semiconductor, respectively. $E_{\mathrm{CB}}$ and $E_{\mathrm{VB}}$ are the energies of the conduction band (CB) and valence band (VB) edges, respectively. $\mathbf{b} \mid \mathrm{A}$ p-n diode junction is the key building block for optoelectronic devices. c | A photovoltaic device uses minority carriers and yields the photocurrent $I_{p h}$ and the photovoltage $V_{\mathrm{ph}}$. d $\mid$ In a light-emitting diode (LED) device, a forward bias $V_{\text {bias }}$ is applied to drive the majority carrier flow. Carrier recombination leads to light emission in direct bandgap semiconductors. $h v$, photon energy. 
a

OD semiconductor

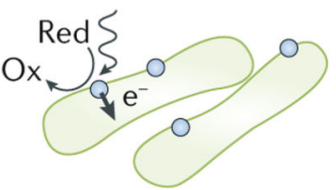

Photosynthesis

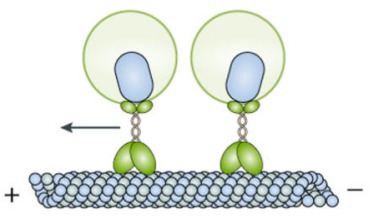

Transport study
1D semiconductor

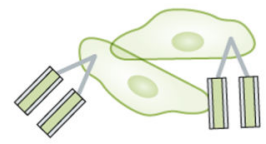

Intracellular electrophysiology

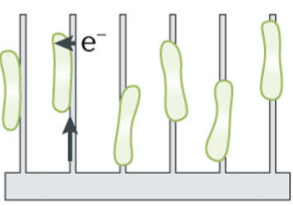

Microbial fuel cell

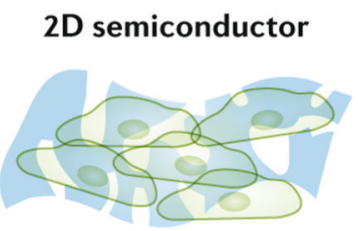

Transient electronics

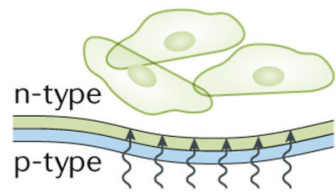

Optoelectronic stimulation d

3D semiconductor

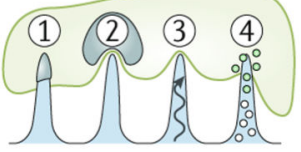

Multifunctional probe

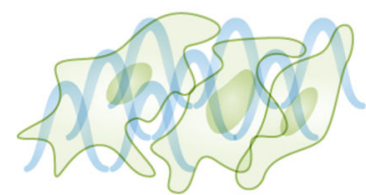

3D sensor network

e

Bioelectronic interfaces

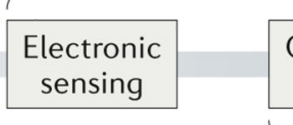
Optoelectronic
sensing Optoelectronic Photothermal Photoluminescence stimulation imaging

Biophotonic interfaces

Fig. 4 |. Semiconductor geometries and possible modes for biointerfaces.

a 1 OD semiconductors can be used to mimic photosynthesis, for example, by using $\mathrm{CdS}$ nanoparticles that are precipitated on the cell wall of a bacterium to sensitize nonphotosynthetic bacteria through photoinduced electron transfer pathways. Photoluminescent quantum dots can be coupled to motor proteins to enable the tracking of intracellular transport mechanisms. b | 1D semiconductors, for example, nanoscale kinked Si nanowire field-effect transistors, allow intracellular recordings of single cell action potentials. Nanowire-bacteria hybrids can photoelectrochemically fix carbon dioxide and produce value-added chemicals. c| $2 \mathrm{D}$ semiconductors, for example, biodegradable $\mathrm{Si}$, provide a physically transient form of electronic devices. The photovoltaic effect of thin-film Si diode junctions can be used for the optical control of biological activities. d |3D semiconductors, for example, semiconductor micropillar or nanopillar arrays, can detect cellular electrophysiological signals, potentially probe nucleus mechanics, deliver optical stimuli for photostimulation and release drugs. Strain-engineered 3D mesostructures of Si can serve as electronic scaffolds for neural networks. e | Typical signal transduction mechanisms of semiconductor devices involve electrical or optical inputs and outputs. Ox, oxidation; Red, reduction. 
a

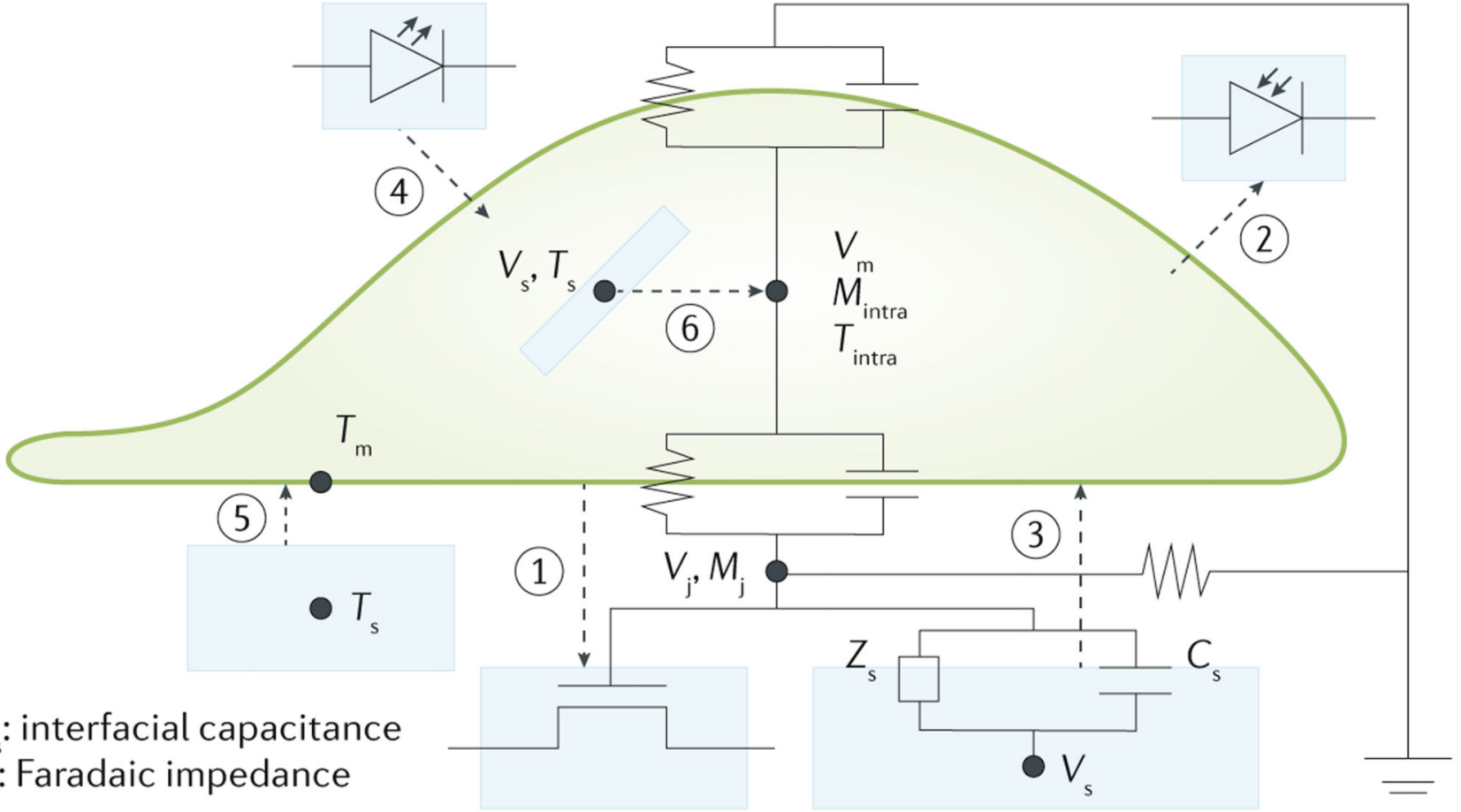

b
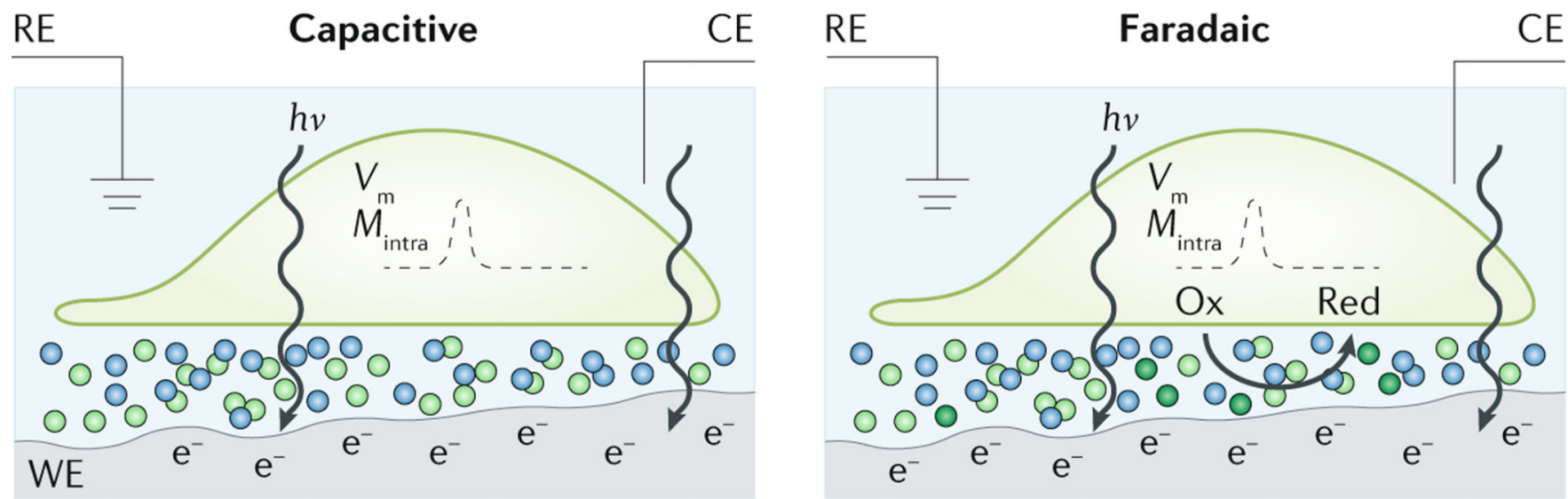

Fig. 5 |. Biophysical mechanisms of signal transduction at semiconductor biointerfaces.

a There are six basic operation modes of semiconductor-enabled biointerfaces. (1) A fieldeffect transistor can sense device-cell junctional bioelectric signals $\left(V_{\mathrm{j}}\right)$ and biomolecule concentrations $\left(M_{\mathrm{j}}\right)$ through its gate-controlled drain-source currents. (2) A photodiode can sense bioluminescence. (3) The photovoltaic effect of a semiconductor can generate a photovoltage $\left(V_{\mathrm{s}}\right)$ upon light illumination, which can locally modulate biological activities through either capacitive $\left(C_{\mathrm{S}}\right)$ or Faradaic $\left(Z_{\mathrm{s}}\right)$ effects. (4) Cells can be stimulated by direct light emission using semiconductors in conjunction with other photosensitization techniques, such as optogenetics. (5) Recombination of photogenerated carriers in a semiconductor can dissipate energies through lattice-vibration-induced heating $\left(T_{\mathrm{s}}\right)$ and increase the temperature ( $\left.T_{\mathrm{m}}\right)$ of the cell membrane to modulate biological activities. (6) A 
nanoscale semiconductor can exhibit either photoelectrochemical or photothermal effect inside a cell to change the intracellular potential $\left(V_{\mathrm{m}}\right)$, the intracellular concentration of biomolecules $\left(M_{\text {intra }}\right)$ and the intracellular temperature $\left(T_{\text {intra }}\right)$. b $\mid$ Light-induced capacitive and Faradaic effects from semiconductor surfaces. Upon light illumination, excessive charge carriers (for example, electrons) accumulate near the semiconductor surface. Subsequently, the charge imbalance at the semiconductor-electrolyte interface triggers counterions (lightgreen spheres) in the electrolyte to migrate to the electrode surface. Inhibition of the charge injection causes the ionic flow to charge only the Helmholtz double layer, resulting in a transient capacitive current. If charges are injected into the solution, light-produced electrons and holes cause the photoelectrochemical reduction (Red) or oxidation (Ox) of solution species (dark-green spheres), that is, cathodic or anodic reactions. In both cases, the transmembrane potentials of local cell membranes $\left(V_{\mathrm{m}}\right)$ and/or concentration of biomolecules $\left(M_{\text {intra }}\right)$ can be altered. WE, CE and RE are working, counter and reference electrodes, respectively, and they are not always required for biointerface studies. The blue, light-green and dark-green spheres represent ions or molecules at the biointerface, but their size and location are not to scale. $h v$, photon energy. 


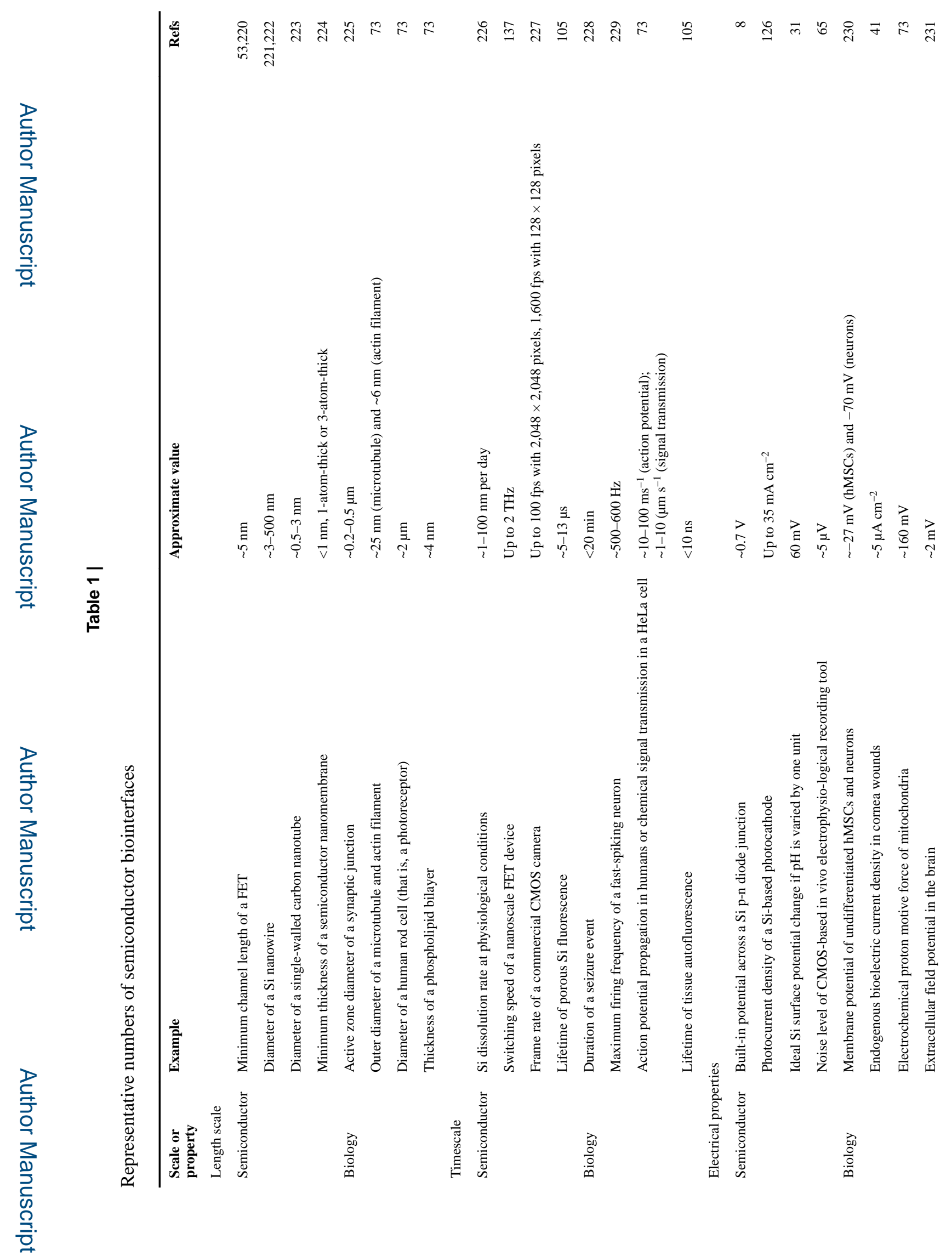

Nat Rev Mater. Author manuscript; available in PMC 2019 October 26. 


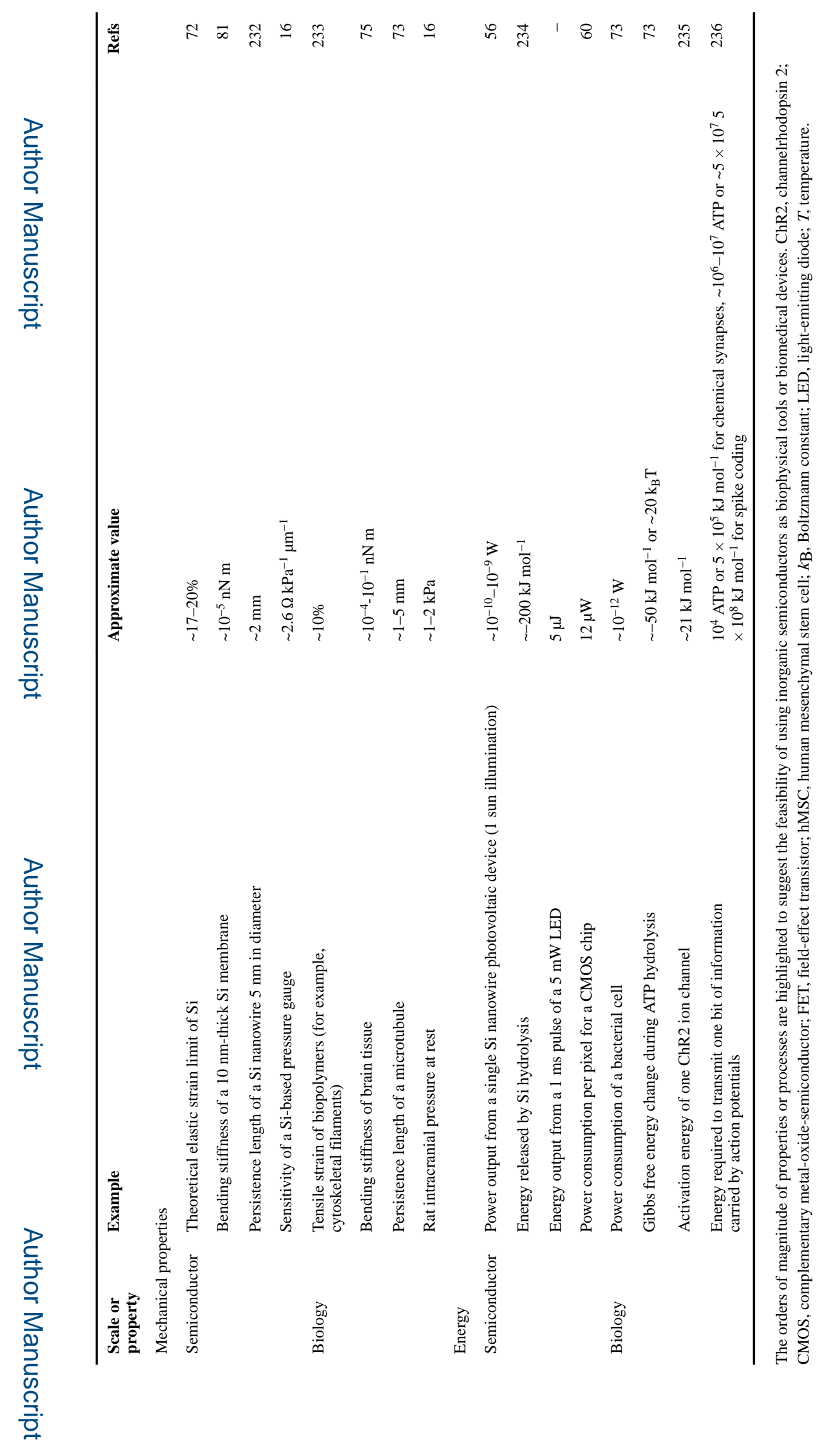

Nat Rev Mater: Author manuscript; available in PMC 2019 October 26. 


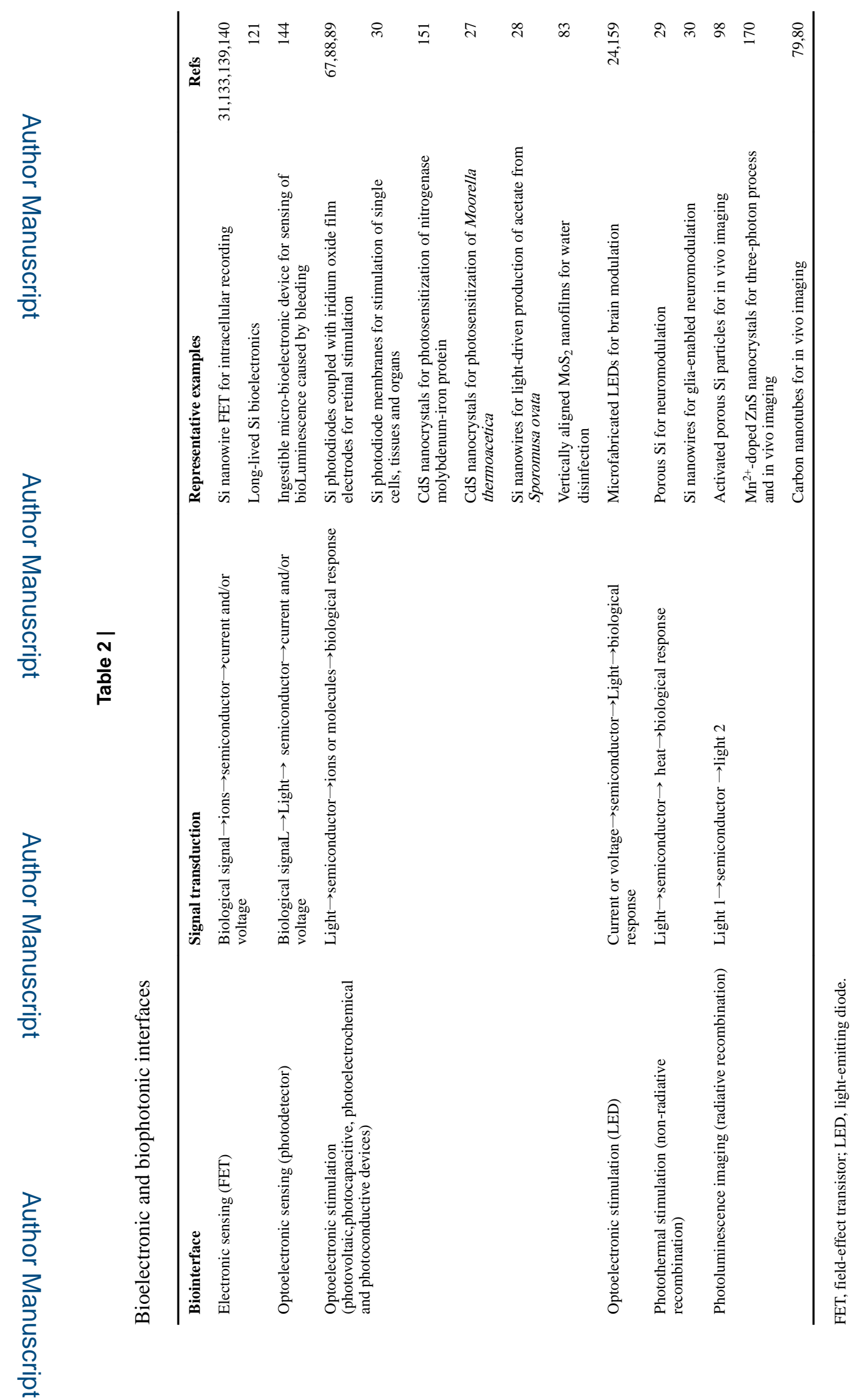

Nat Rev Mater. Author manuscript; available in PMC 2019 October 26. 